\title{
Drosophila Psidin Regulates Olfactory Neuron Number and Axon Targeting through Two Distinct Molecular Mechanisms
}

\author{
Daniel Stephan, ${ }^{1}$ Natalia Sánchez-Soriano, ${ }^{2}$ Laura F. Loschek, ${ }^{1}$ Ramona Gerhards, ${ }^{1}$ Susanne Gutmann, ${ }^{3}$ \\ Zuzana Storchova, ${ }^{3}$ Andreas Prokop, ${ }^{2}$ and Ilona C. Grunwald Kadow ${ }^{1}$ \\ ${ }^{1}$ Sensory Neurogenetics Research Group, Max Planck Institute of Neurobiology, 82152 Martinsried, Germany, ${ }^{2}$ Faculty of Life Sciences, The University of \\ Manchester, Manchester M13 9PT, United Kingdom, and ${ }^{3}$ Maintenance of Genome Stability Research Group, Max Planck Institute of Biochemistry, 82152 \\ Martinsried, Germany
}

The formation of neuronal circuits is a key process of development, laying foundations for behavior. The cellular mechanisms regulating circuit development are not fully understood. Here, we reveal Psidin as an intracellular regulator of Drosophila olfactory system formation. We show that Psidin is required in several classes of olfactory receptor neurons (ORNs) for survival and subsequently for axon guidance. During axon guidance, Psidin functions as an actin regulator and antagonist of Tropomyosin. Accordingly, Psidin-deficient primary neurons in culture display growth cones with significantly smaller lamellipodia. This lamellipodial phenotype, as well as the mistargeting defects in vivo, is suppressed by parallel removal of Tropomyosin. In contrast, Psidin functions as the noncatalytic subunit of the $N$-acetyltransferase complex B (NatB) to maintain the number of ORNs. Psidin physically binds the catalytic NatB subunit CG14222 (dNAA20) and functionally interacts with it in vivo. We define the dNAA20 interaction domain within Psidin and identify a conserved serine as a candidate for phosphorylation-mediated regulation of NatB complex formation. A phosphomimetic mutation of this serine showed severely reduced binding to dNAA20 in vitro. In vivo, it fully rescued the targeting defect but not the reduction in neuron numbers. In addition, we show that a different amino acid point mutation shows exactly the opposite effect by rescuing only the cell number but not the axon targeting defect. Together, our data suggest that Psidin plays two independent developmental roles via the acquisition of separate signaling pathways, both of which contribute to the formation of olfactory circuits.

\section{Introduction}

The development of functional nervous systems relies on the formation of appropriate synaptic connections. The specification of the right number and type of different neurons and the guidance of their axons to their target cells are essential in this process. During targeting, growth cones at axon tips provide a dynamic platform for integration of attractive and repulsive guidance cues (Lowery and Van Vactor, 2009). Through binding to membrane receptors displayed on growth cones, guidance cues orchestrate

Received July 2, 2012; revised Sept. 5, 2012; accepted Sept. 6, 2012.

Author contributions: D.S. and I.C.G.K. designed research; D.S., N.S.-S., L.F.L., R.G., S.G., and I.C.G.K. performed research; I.C.G.K. contributed unpublished reagents/analytic tools; D.S., N.S.-S., L.F.L., R.G., Z.S., A.P., and I.C.G.K. analyzed data; D.S., A.P., and I.C.G.K. wrote the paper.

This work was supported by an Emmy-Noether Grant from the German Research Foundation, by a career development award from the Human Frontiers Science Organization, and by the Max-Planck Society (I.C.G.K.). The work of N.S.-S. and A.P. was funded by the BBSRC (BB/I002448/1) and funds by the Wellcome Trust supporting the Manchester Fly Facility (087742/Z/08/Z). We are very grateful to $D$. Montell for sharing data and reagents before publication. We thank V. Schilling and C. Knappmeyer for great technical help. The psidin ${ }^{16978}$ allele was found by I.C.G.K. during her postdoctoral stay in the laboratory of S. L. Zipursky. We thank S. L. Zipursky and Pelin C. Volkan for fruitful collaborations and discussions. Budding yeast strain lacking MDM20 was kindly provided by Dr. F. S. Sherman (University of Rochester Medical Center, Rochester, NY).

The authors declare no competing financial interests.

Correspondence should be addressed to llona C. Grunwald Kadow, Max Planck Institute of Neurobiology, Sensory Neurogenetics Research Group, Am Klopferspitz 18, 82152 Martinsried, Germany. E-mail: ikadow@neuro.mpg.de. DOI:10.1523/JNEUROSCI.3116-12.2012

Copyright $\odot 2012$ the authors $\quad 0270-6474 / 12 / 3216080-15 \$ 15.00 / 0$ dynamic changes of the cytoskeleton, in particular of the actin and microtubule networks (Driessens et al., 2001; GordonWeeks, 2004; Kalil and Dent, 2005; Evans et al., 2007; Lowery and Van Vactor, 2009). The growth cone consists of actin-rich structures, finger-like filopodia, and veil-like lamellipodia. Dynamic changes of the actin networks are mediated by actin-binding proteins, such as disassembly factors (e.g., cofilin) and stabilizers (e.g., Tropomyosin, Fascin) (Pak et al., 2008). Their activity is regulated through common effectors of signaling events involved in pathfinding (Huber et al., 2003; Ng and Luo, 2004; Hall and Lalli, 2010). However, how these molecular pathways operate and are integrated at the growth cone level to implement predictable morphogenetic changes is not fully understood.

To gain this understanding, the olfactory system of Drosophila provides an efficient experimental platform to study different aspects of neuronal specification, axon guidance, and synapse formation (Rodrigues and Hummel, 2008; Brochtrup and Hummel, 2011). Two peripheral olfactory organs, the third segment of the antenna and the maxillary palp (MP), contain olfactory receptor neurons (ORNs) located mostly in groups of two to four neurons inside chemosensory sensilla. ORNs develop from the eye-antennal disc in a series of asymmetric divisions and regulated cell death to reach their final sensilla type-dependent composition of different ORN classes plus several kinds of supporting cells (Rodrigues and Hummel, 2008). All ORNs project through 
A

A
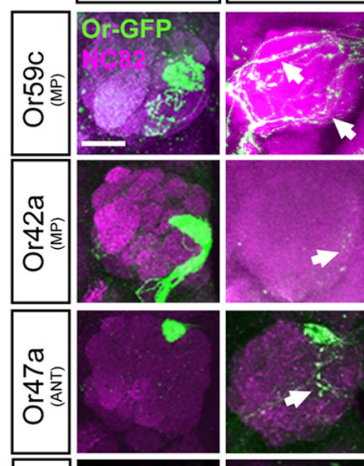

ฟูป

forward eyFIp MARCM

C

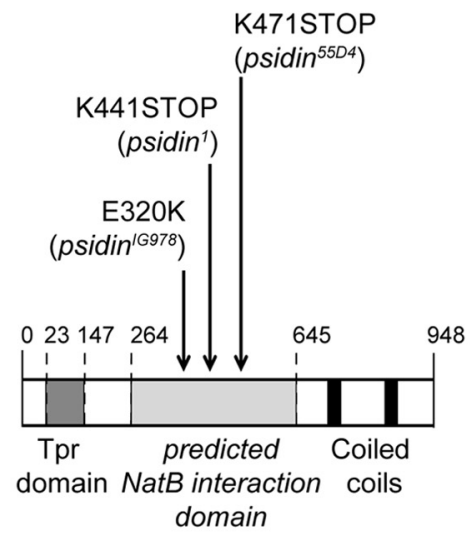

psidin $^{5504}$
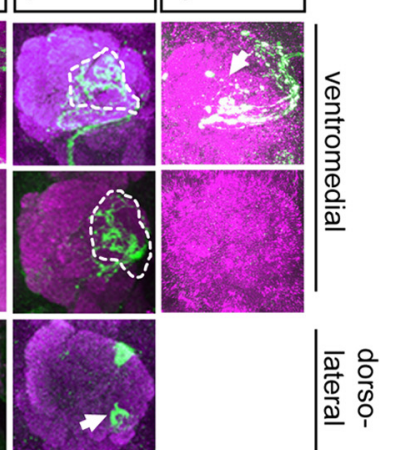

B

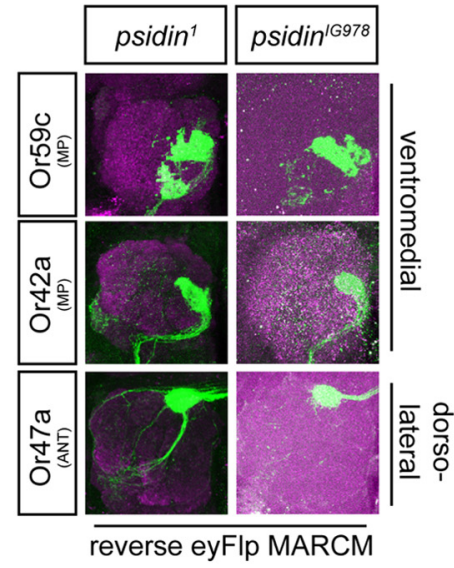

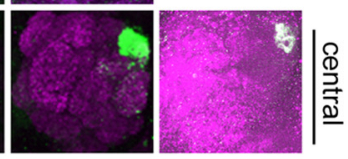
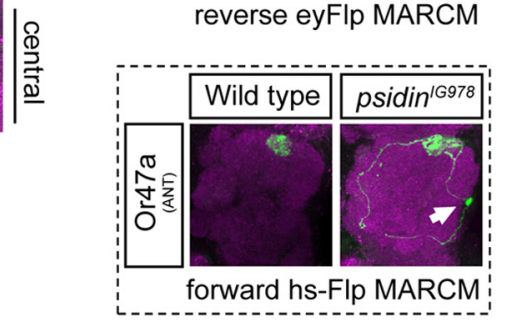

D

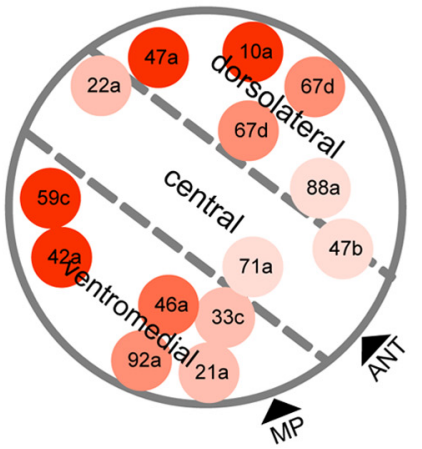

$0 \%$ targeting phenotype $100 \%$
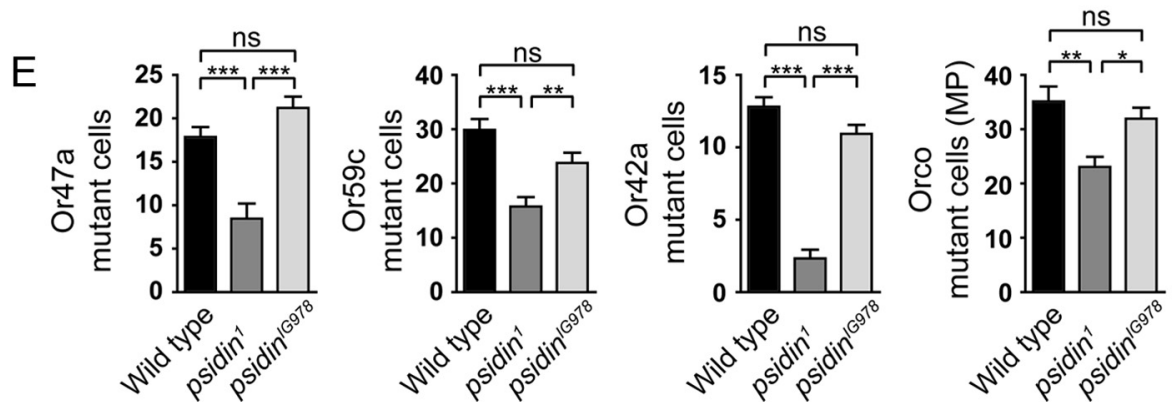

Figure 1. Psidin is required for pathfinding of a subset of olfactory receptor neurons. Adult brains were stained with anti-GFP (green) and NC82 (magenta) to visualize synaptic innervation of ORNs in the AL. In forward MARCM, only mutant cells are visible selectively labeled in green in a wild-type background. Conversely, wild-type cells are labeled in a mutant background in reverse MARCM. MARCM analysis was done using eyFlp or hsFlp to generate clones. $A, 0$, $59 \mathrm{c}$ and Or42a mutant axons show a strong defasciculation phenotype in the psidin $^{7}$ and psidin ${ }^{5504}$ background (dashed lines). Or47a psidin mutant axons show ectopic synapse formation in ventral parts of the AL (arrows). (entrally projecting 0 r22a mutant

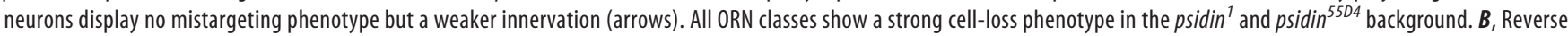
MARCM analysis of eyFlp clones. Or59c, Or42a, and Or47a wild-type neurons project normally in the psidin ${ }^{7}$ or psidin ${ }^{16978}$ mutant background. Forward MARCM analysis using hsFlp showed that small psidin ${ }^{16978}$ clones are sufficient to cause mistargeting in Or47a axons. C, Protein structure of Psidin and the used psidin alleles. Psidin contains a Tpr-domain and coiled coils at the C terminus. A "NatB interaction domain" is predicted in the center of Psidin. D, ORN classes are affected differently in targeting according to their projection route in psidin" mutants. $E$, Mutant cells in the adult ANT and MP were counted using 0r-gal4 and UAS-mCD8-GFP. The number of neurons is significantly reduced in the psidin ${ }^{7}$ loss-of-function mutant background in all classes. Although the neuron number is not significantly reduced in psidin ${ }^{16978}$ mutants, there is a trend toward a reduction. Counting of Or83b-positive cells (Orco) in the MP revealed a $35 \%$ reduction of neurons in the $p$ sidin ${ }^{7}$ background. ${ }^{*} p<0.05 ;{ }^{* *} p<0.01 ;{ }^{* * *} p<0.001$, one-way ANOVA and Bonferroni's posttest. Error bars \pm SEM. Scale bar, $20 \mu \mathrm{m}$. 
the antennal or labial nerves into the antennal lobe $(\mathrm{AL})$ in the brain, where they form stereotypic synaptic contacts. Each ORN expresses the general olfactory receptor named ORCO (Or83b) together with a specific odorant-binding receptor (OR) out of a set of $\sim 60$ genes (Rodrigues and Hummel, 2008; Vosshall and Hansson, 2011). ORNs expressing the same OR innervate the same glomerulus, a structural AL sub-compartment, where they form synaptic contacts with local interneurons and projection neurons (PN) at stereotypic positions. This innervation pattern relies on a complex hierarchical guidance process using ORN axon-axon interactions and axon-target interactions (ORN-PN) (Brochtrup and Hummel, 2011).

We identified the actin-binding protein Psidin (Brennan et al., 2007; Kim et al., 2011) as a novel regulator of olfactory system development. We provide in vitro and in vivo evidence that Psidin is required during two independent steps of circuit development: as a component of the $N$-acetyltransferase complex B (NatB) complex to maintain neuron number and as an actin-binding protein during axonal targeting. During targeting, we suggest that Psidin influences growth cone responsiveness to guidance cues by regulating actin dynamics. In the olfactory system, this translates into a class-specific Psidin requirement correlating with the specific routes and distances the ORN growth cone travels.

\section{Materials and Methods}

\section{Fly genetics}

Fly stocks were raised at $25^{\circ} \mathrm{C}$ on standard cornmeal medium. The ethyl methanesulfonate (EMS) screen was performed as described previously (Cayirlioglu et al., 2008). ORN analysis was performed using the MARCM (mosaic analysis with a repressible cell marker) technique with flies of the following genotype: Or-GAL4 UAS-syt-GFP (or UAS-mCD8GFP)/+; FRT82 mutation/FRT82 gal80 (E2F). ORNs were labeled using GAL4 or direct fusion of the respective OR promoter elements. All analyses were done in eyFlp mosaic animals. The following alleles of Psidin were used: $\operatorname{sidin}^{I G 978}$ (this study), psidin ${ }^{1}$ (Brennan et al., 2007), and psidin ${ }^{55 D 4}$ (Kim et al., 2011). All psidin mutations were lethal and noncomplementing. Act-GAL4 was used to drive the expression of various UAS elements in eyFlp clones. UAS-Psidin-HA was generated and generously provided by D. Montell (Johns Hopkins School of Medicine, Baltimore, MD). Transgenic flies carrying RNAi against dNAA20 were ordered from the Kyoto and Vienna Drosophila RNAi Center (VDRC) stock centers. Flies carrying overexpression constructs of LimK and Cofilin were obtained from the Bloomington Stock Center. Three different RNAi lines [VDRC (lines 1 and 2), NIG-Kyoto (line 2)] were used to knock down dNAA20. We saw similar results for all three lines.

Detailed genotypes. For all genotypes, X stands for FRT82B, FRT82B psidin ${ }^{I G 978}$, FRT82B psidin ${ }^{55 D 4}$, or FRT82B psidin ${ }^{1}$.

MARCM genotypes. eyflp; Or-GAL4 UAS-syt-GFP (or UAS-mCD8GFP)/+; FRT82,CL,gal80/X.

Reverse MARCM genotypes. eyflp; Or-GAL4 UAS-syt-GFP (or UASmCD8-GFP)/+; FRT82/X gal80.

Rescue experiment. eyflp; Or59c-mCD8-GFP,act-gal4/+; FRT82,CL, gal80/X, eyflp; Or59c-mCD8-GFP,act-gal4/UAS-Psidin-HA; FRT82,CL, gal80/X, eyflp; Or59c-mCD8-GFP,act-gal4/UAS-p35; FRT82,CL,gal80/X,

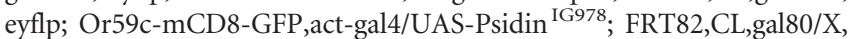
eyflp; Or59c-mCD8-GFP, act-gal4/UAS-Psidin ${ }^{\text {S678A; FRT82,CL,gal80/ }}$

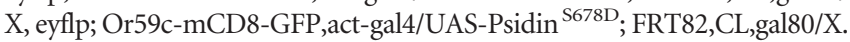

Tropomyosin experiment. eyflp; Or59c-mCD8-GFP/+; FRT82,CL,gal80/ FRT82 psidin ${ }^{1}$, eyflp; Or59c-mCD8-GFP/+; FRT82,CL,gal80/FRT82 psidin ${ }^{5504}$, eyflp; Or59c-mCD8-GFP/+; FRT82,CL,gal80/

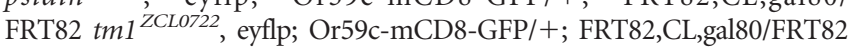
tm1 ${ }^{\text {ZCLO722 }}$ psidin $^{55 D 4}$.

RNAiexperiments. eyflp; Or59c-mCD8-GFP,act-gal4/UAS-RNAi;FRT82, CL,gal80/X, eyflp; Or42a-mCD8-GFP,act-gal4/UAS-RNAi; FRT82,CL, gal80/X, eyflp; Or59c-mCD8-GFP,act-gal4/+; FRT82,CL,gal80/X, eyflp; Or42a-mCD8-GFP,act-gal4/+; FRT82,CL,gal80/X.

Overexpression experiments. eyflp; Or59c-mCD8-GFP,act-gal4/ UAS-Limk(wt); FRT82,CL,gal80/X, eyflp; Or59c-mCD8-GFP,act-gal4/
Table 1. Quantification of the targeting and neuron-loss phenotypes

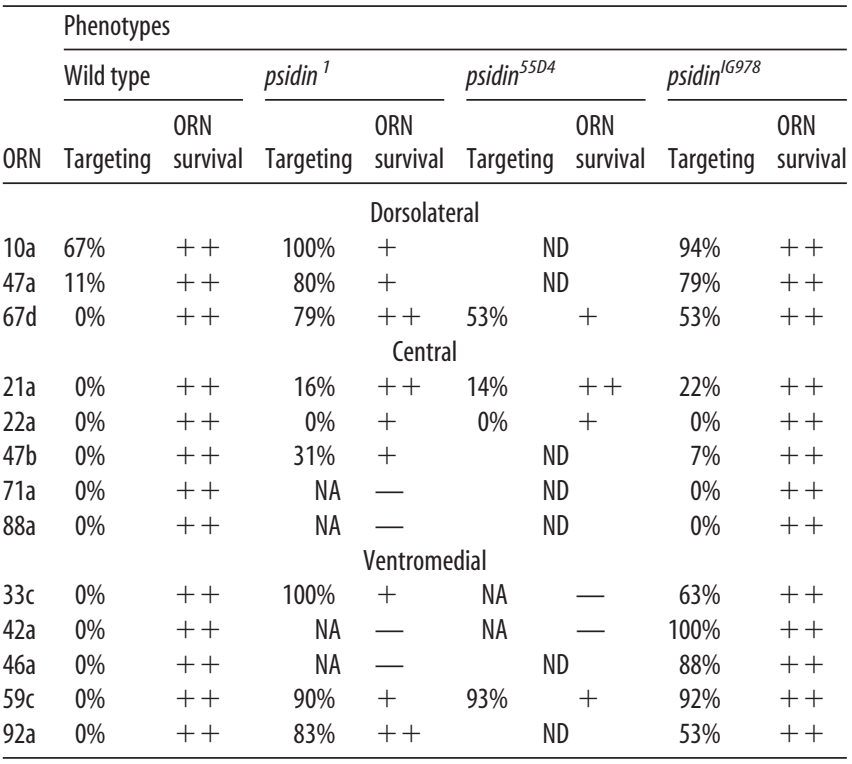

The targeting phenotype was quantified using MARCM analysis of the respective OR marker. Survival of ORNs was scored in three categories: ++ , full survival; + , mild cell loss; - , complete loss of cells; ND, Not determined; NA, not applicable (due to $100 \%$ cell loss).

UAS-Limk(KI);FRT82,CL,gal80/X,eyflp;Or59c-mCD8-GFP,act-gal4/UASLimk(RNAi); FRT82,CL,gal80/X, eyflp/UAS-TsrS3A; Or59a-mCD8-GFP, act-gal4/+; FRT82,CL,gal80/X, eyflp/UAS-TsrS3E; Or59a-mCD8-GFP,actgal4/+; FRT82,CL,gal80/X, eyflp; Or59c-mCD8-GFP,act-gal4/+; FRT82,CL,gal80/X.

\section{Histology}

Adult fly brains were dissected to analyze the ORN targeting pattern as described previously (Hartl et al., 2011). For each experiment, a minimum of 20 brains from male and female flies were analyzed per condition (i.e., $n=20-36$ ). Pupal stages were determined by collecting white pupae and incubating them at $25^{\circ} \mathrm{C}$ until they reached the correct age. The cell numbers of ORNs were determined in adult antennae (ANTs) and MPs. Confocal pictures were taken using the Olympus FV1000 and Leica SP2. Images were processed in ImageJ and Adobe Photoshop.

\section{In situ hybridization}

A $671 \mathrm{bp}$ fragment (antisense probe) and a $682 \mathrm{bp}$ fragment (sense probe) of psidin cDNA (GenBank accession number LD30731) was cloned into the pCRIITOPO vector. Hybridization was performed at $55^{\circ} \mathrm{C}$ using standard methods (Green and Sambrook, 2012). Probes were detected using anti-DIG antibody coupled to alkaline phosphatase (Roche). Staining was developed with NBT/BCIP substrate. Pictures were taken using the Leica MZ500.

\section{Quantification of targeting phenotype}

We used three different categories to describe the phenotype of Or59c neurons. Normal wild-type targeting displays a strongly innervated Or59c glomerulus and no additional innervation on the dorsal area of the AL. Mild mistargeting shows a weaker innervation of the glomerulus and additional mistargeting and axon dispersal across the AL. Strong mistargeting was scored when there was no concentrated innervation in the Or59c target glomerulus, but rather random innervations across the entire AL. Statistical analysis was done using one-way ANOVA and Bonferroni's posttest $\left({ }^{\star} p<\right.$ $\left.0.05 ;{ }^{* *} p<0.01 ;{ }^{* * *} p<0.001\right)$. Error bars depict the SEM.

\section{Coimmunoprecipitation experiments}

S2 cells were transfected using Effectene (QIAGEN). Ubiquitin-GAL4 (ub-GAL4) was used to drive the expression of UAS-Psidin-HA, UASPsidin ${ }^{\text {G978 }}$-HA, UAS-Psidin ${ }^{\text {S678X }}{ }_{-H A}$, UAS-Psidin ${ }^{\triangle N_{a t B X}}$-HA, and UAS-dNAA20-myc. All constructs were N-terminally tagged with HA or myc. Cells were harvested after $3 \mathrm{~d}$ and lysed in lysis buffer [50 mM Tris, pH 7.4, $150 \mathrm{~mm} \mathrm{NaCl}, 2$ mм EDTA, $1 \%$ Triton X-100, and protease 

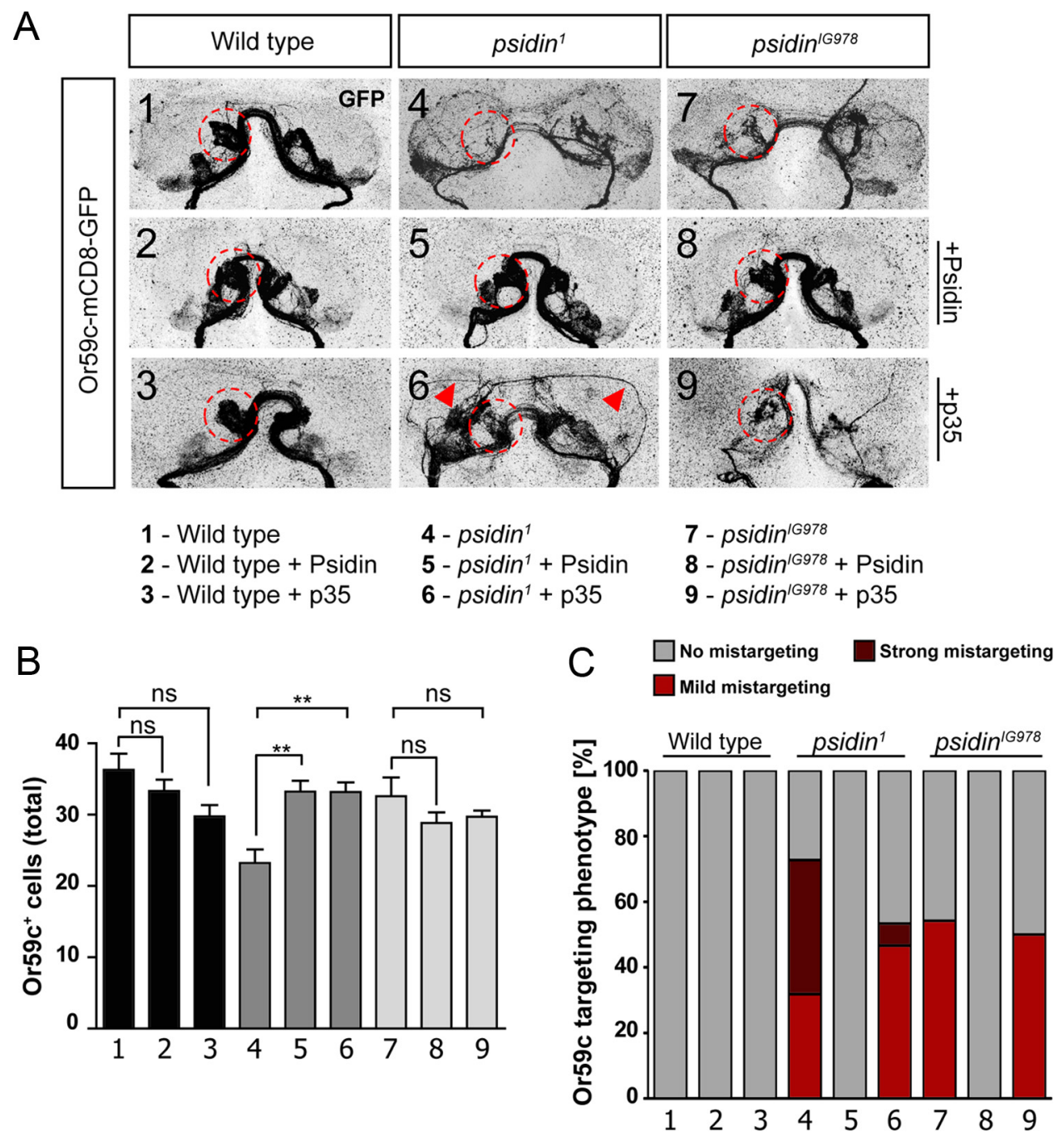

Figure 2. Cell-loss phenotype can be rescued independent from the targeting phenotype. The entire ORN population was visualized using a direct-fusion Or-mCD8-GFP. Act-GAL4 was used to drive the expression of wild-type Psidin or p35 in eyFlp clones. A, A mistargeting phenotype is visible in both the psidin ${ }^{16978}$ and psidin ${ }^{7}$ mutant backgrounds, displaying mildly and strongly dispersed (dashed circles) glomerular innervation, respectively. Additionally, in the psidin ${ }^{1}$ background, axons mistarget to dorsal parts of the AL (arrowheads). The normal targeting pattern is restored after reexpression of wild-type Psidin in the respective mutant background. In contrast, expression of $\mathrm{p} 35$ does not rescue the mistargeting phenotype. $\boldsymbol{B}$, We observed a strong loss of neurons in the psidin ${ }^{7}$ background. In the psidin ${ }^{7}$ background, the ORN number was reduced by $36 \%$ compared with wild type. Reexpression of wild-type Psidin rescues the ORN cell number in the psidin ${ }^{7}$ background. Similarly, expression of $\mathrm{p} 35$ restores the neuron number in the psidin loss-of-function background. The neuron number was not significantly changed in the psidin ${ }^{16978}$ background. $C$, Quantification of the mistargeting phenotype. In the psidin ${ }^{7}$ mutant background, 41 and $32 \%$ of the neurons display a strong or mild mistargeting phenotype, respectively. Mistargeting phenotype is completely rescued after expression of wild-type Psidin. Expression of p35 reduces the targeting phenotype only to levels comparable to psidin ${ }^{/ 6978}$ mutants. Similarly, in the $p s i d i n^{1 / 6978}$ background, reexpression of wild-type Psidin rescues the phenotype completely. ${ }^{*} p<0.05 ;{ }^{* *} p<0.01$; ${ }^{* *} p<0.001$, one-way ANOVA and Bonferroni's posttest. Error bars \pm SEM.

inhibitor (Sigma-Aldrich)]. Lysate was cleared, and supernatant was used for coimmunoprecipitation. Proteins were incubated with rat anti-HA (1:1000; Sigma-Aldrich) and Sepharose beads [50\% (v/v)] for $2 \mathrm{~h}$ at $4^{\circ} \mathrm{C}$. Afterward, samples were boiled in SDS loading buffer and analyzed using Western blot. dNAA20 cDNA from clone LD30731 was subcloned into a pUAST vector and fused to a myc tag. Five repetitions were performed for each condition.

\section{Western blot quantification}

Western blots were quantified using ImageJ. The ratio of dNAA20 to Psidin ${ }^{\mathrm{X}}$ was quantified measuring the intensity of the respective bands. We compared the amount of dNAA20 pulled down with wild-type Psidin versus the amount of dNAA20 pulled down with a Psidin variant. We measured the respective bands of HA-captured Psidin and pulled-down dNAA20. Only the HA-bound fraction of Psidin can effectively pull down dNAA20. The same was done for wild-type Psidin and dNAA20, which was used as a reference on each blot. We used the wild-type control to normalize the pulldown of dNAA20 with a Psidin variant on each individual blot.

\section{RNAi knock down in $\mathrm{S} 2$ cell culture}

S2 cells were transfected using Effectene (QIAGEN). Ub-GAL4 was used to drive the expression of the RNAi against dNAA20 and UAS-dNAA20myc. Cells were harvested after $3 \mathrm{~d}$ and lysed in lysis buffer [50 mM Tris, $\mathrm{pH}$ 7.4, $150 \mathrm{~mm} \mathrm{NaCl}, 2 \mathrm{~mm}$ EDTA, 1\% Triton X-100, and protease inhibitor (Sigma-Aldrich)]. Protein samples were loaded on SDS gels and detected with anti-tubulin and anti-myc antibodies. Western blots were quantified by measuring the intensity of the tubulin loading control and the amount of Psidin in each lane using ImageJ software. Experimental bands (anti-myc band) of one lane were normalized to their respective tubulin control of the same lane. A single transfected lane (UASdNAA20-myc only) was used as the control for RNAi efficiency. Each condition was repeated four times.

\section{Generation of Psidin isoforms}

Psidin ${ }^{\text {G } 978}$, Psidin ${ }^{\text {S678A }}$, and Psidin ${ }^{\text {S678D }}$ were generated using the QuikChange Lightning Site-Directed Mutagenesis kit (Agilent Technologies). Wild-type UAS-Psidin-HA ( provided by D. Montell) was used as 


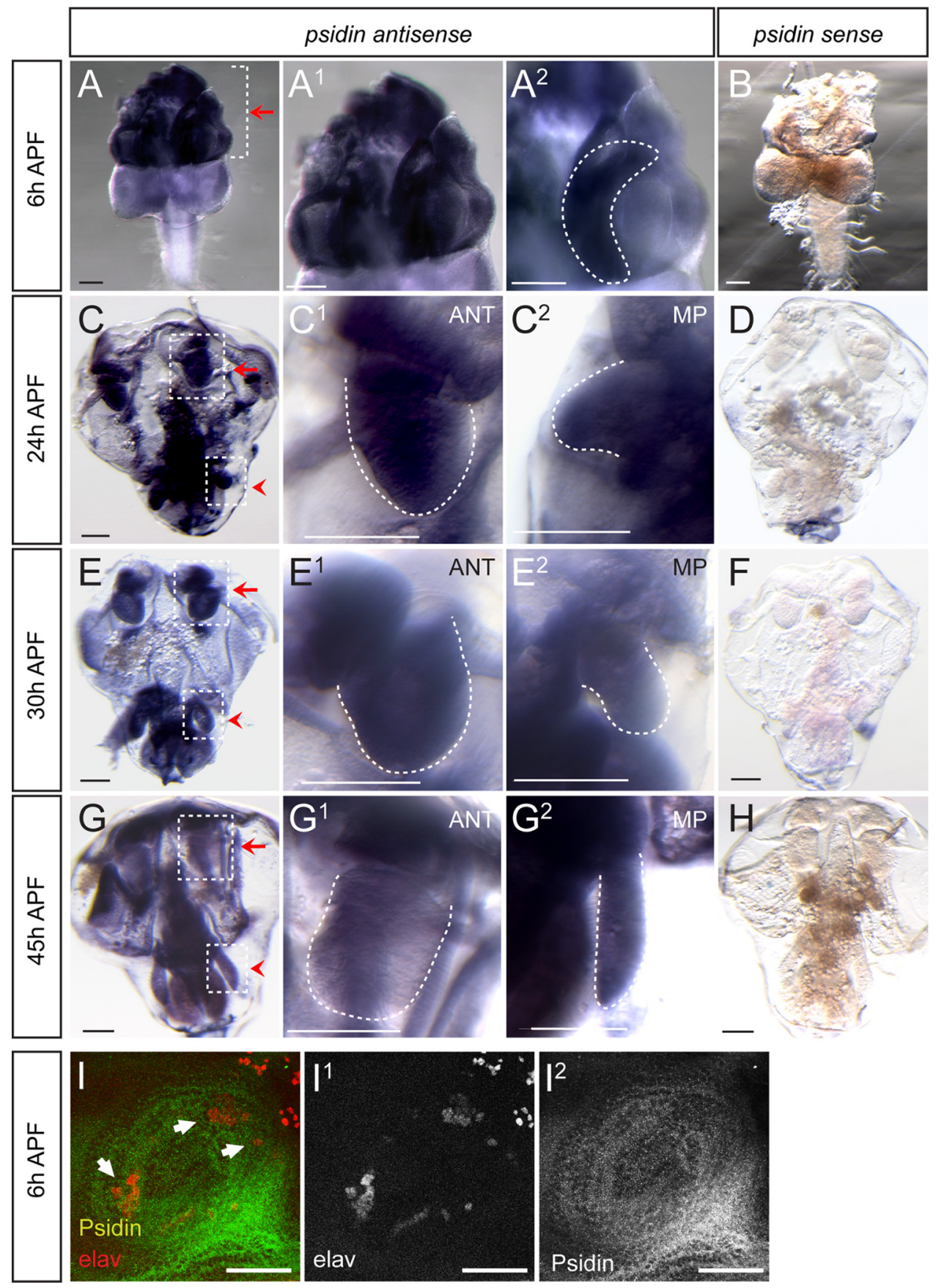

Figure 3. Psidin is expressed in the developing olfactory organs. $\boldsymbol{A}-\boldsymbol{H}, \mathrm{In}$ situ hybridization (ISH) for psidin at different developmental stages. A, At $6 \mathrm{~h}$ APF, the psidin ISH signal can be detected in the developing eye-antennal discs (arrow). C, E, The expression stays high in the developing antenna and MPs both at 24 and $30 \mathrm{~h}$ APF (arrows). G, Psidin expression starts to decline at $45 \mathrm{~h}$ APF. $B, D, F, H$, Control (sense) probes for psidin do not show any signals. $I$, Staining of a developing antennal disc in $6 \mathrm{~h}$ APF pupae. Psidin is widely expressed in the developing antenna at this stage and partially correlates with elav-positive cells. Scale bars, $100 \mu \mathrm{m}$.

a template. Psidin ${ }^{\Delta N a t B X}$ constructs were generated using PCR-mediated generation of deletions.

\section{Drosophila primary neuron cultures}

Drosophila primary neuron cultures were generated as described previously (Sánchez-Soriano et al., 2010). In brief, cells were removed with micromanipulator-attached capillaries from stage 11 embryos $\left(6-7 \mathrm{~h}\right.$ after egg laying at $\left.25^{\circ} \mathrm{C}\right)$ (Campos-Ortega and Hartenstein, 1997), treated for $5 \mathrm{~min}$ at $37^{\circ} \mathrm{C}$ with dispersion medium, washed, and dissolved in 30-40 $\mathrm{ml}$ of Schneider medium (Schneider, 1964). Then, the aliquots were transferred to coverslips and kept as hanging drop cultures in air-tight, special culture chambers (Dübendorfer and Eichenberger-Glinz, 1980) usually for $6 \mathrm{~h}$ at $26^{\circ} \mathrm{C}$. Cultured Drosophila neurons were analyzed $6 \mathrm{~h}$ after plating. They were fixed (30 min in $4 \%$ paraformaldehyde $/ 0.05 \mathrm{M}$ phosphate buffer, $\mathrm{pH}$ 7.2) and washed in PBS and 0.1\% Triton X-100 (PBT). Incubation with antibodies was performed in PBT. Microtubules were stained with anti-tubulin (1:1000; Sigma-Aldrich) and FITC- or Cy3conjugated secondary antibodies (1:200; Jackson ImmunoResearch). Filamentous actin was detected with TRITC-conjugated phalloidin (Sigma-Aldrich). Stained Drosophila neurons were mounted in Vectashield (Vector Laboratories). Primary neuron culture images were taken using an AxioCam camera mounted on an BX50WI microscope (Olympus). Lamellipodia area was quantified using ImageJ. Statistical analyses were performed with SigmaStat software using a $t$ test or MannWhitney rank sum test. 

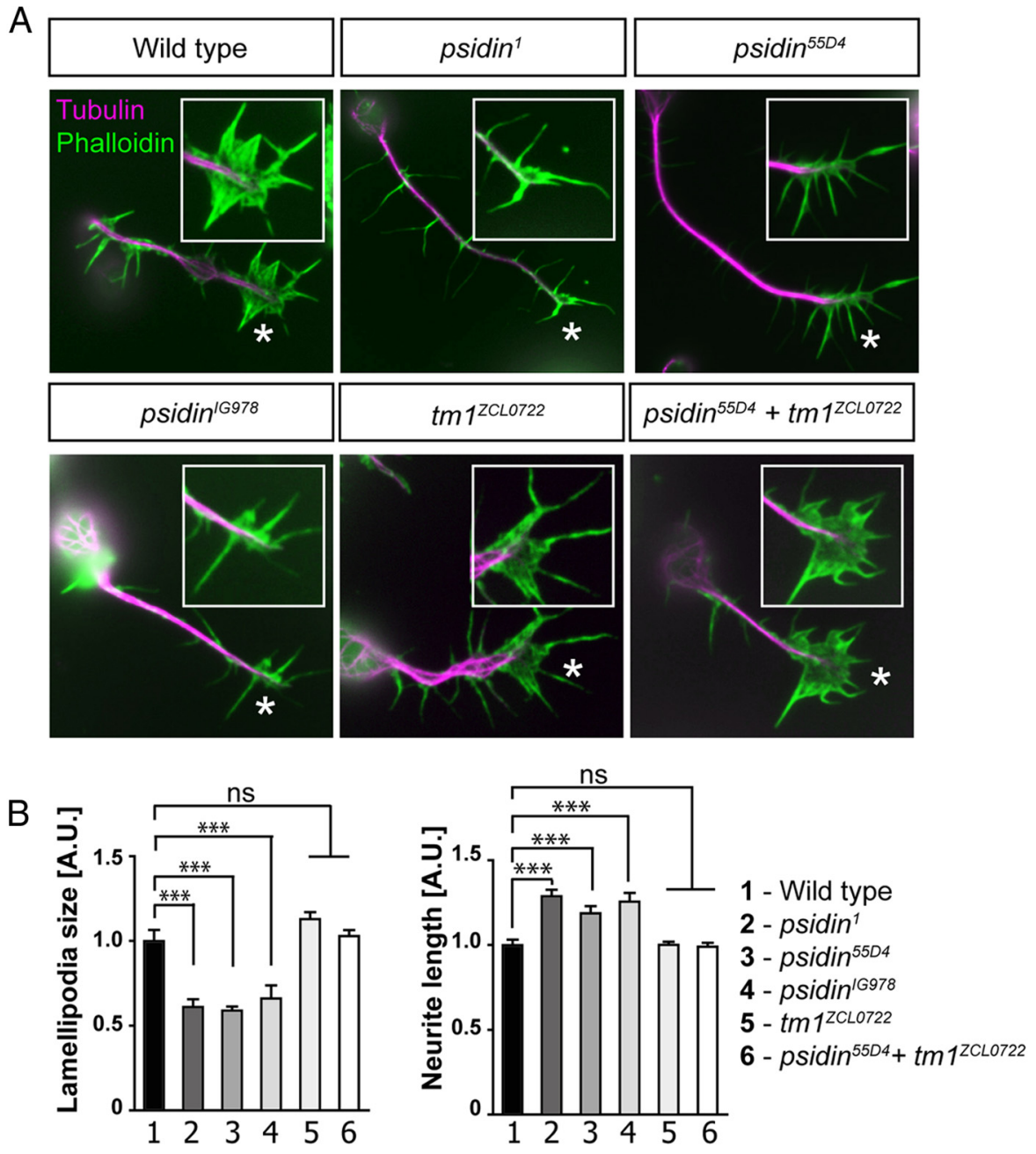

C
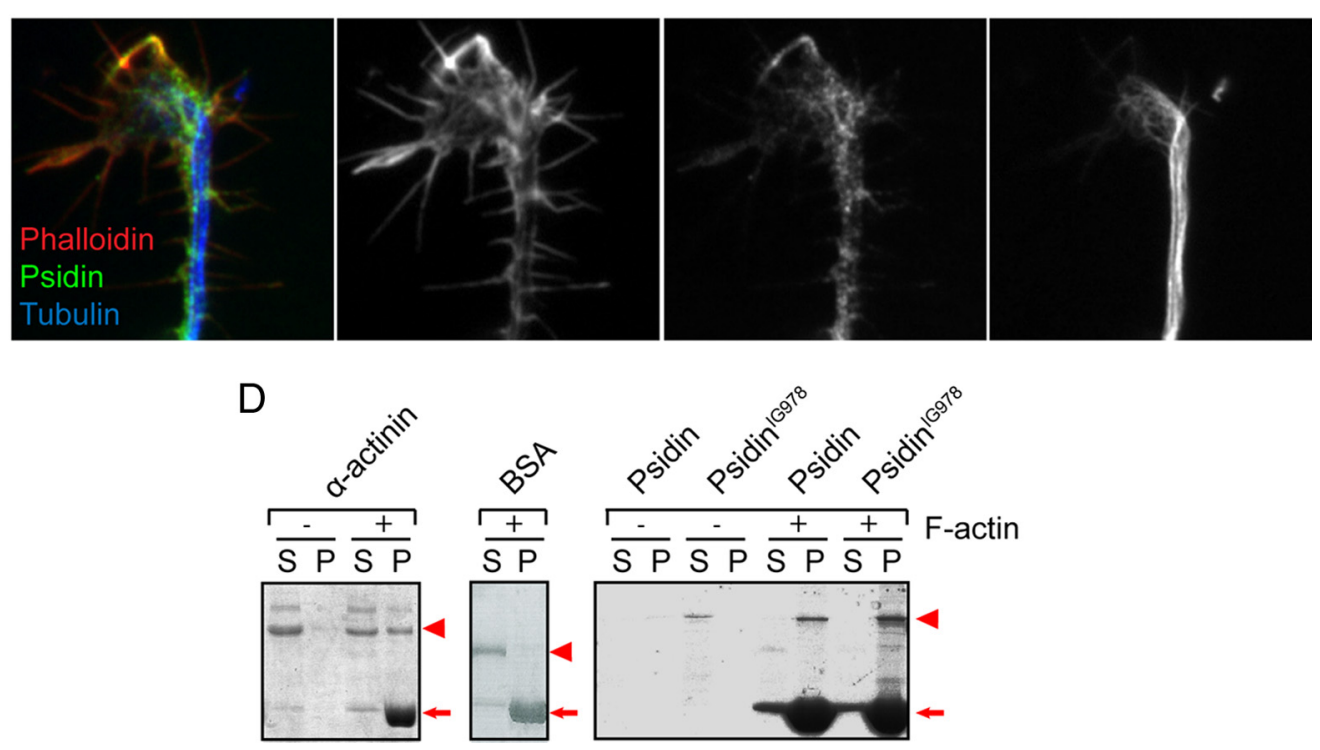

Figure4. Psidin regulateslamellipodiasizeinthegrowthcones. $A, B$, Growth cones (asterisks, $A$ ) aresignificantlysmallerin psidin ${ }^{1}$, psidin ${ }^{504}$, and psidin ${ }^{16978}$ neurons compared with wildtype.Specifically, neurons lacking

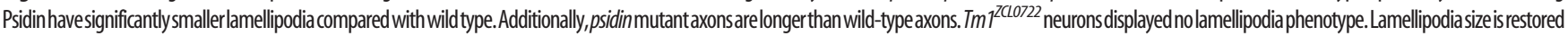
in the psidin ${ }^{504}$ plus $t m 7^{Z 2 C 10722}$ double mutants. C, Primary culture of 6-h-old neurons from wild-type embryos overexpressing HA-tagged Psidin. It localizes to the growth cone together with actin and is visible in the lamellipodia. Neurons were stained with anti-tubulin, phalloidin, and anti-HA (Psidin). D, F-actin pelleting assay. Arrows indicate F-actin, and arrowheads represent the respective proteins: $\alpha$-actinin, BSA, Psidin, and Psidin ${ }^{16978}$. The indicated proteins were incubated in the presence $(+)$ and absence $(-)$ ofF-actin. Reaction mixtures were incubated and centrifuged at $150,000 \times g$. Supernatant $(S)$ and pellet $(P)$ were then separately loaded onto an SDS gel. Thepositive control, $\alpha$-actinin, can befound in thepellettogetherwithF-actin. In contrast, thenegativecontrol, BSA, can only befound in thesupernatant. Both, wild-typePsidin and Psidin ${ }^{16978}$ are only found in the pellet in the presence ofF-actin. Both proteins seem to pellet with F-actin at comparable levels. ${ }^{*} p<0.05 ;{ }^{* *} p<0.01 ;{ }^{* * *} p<0.001$, one-way ANOVA and Bonferroni's posttest. Error bars \pm SEM. 
A
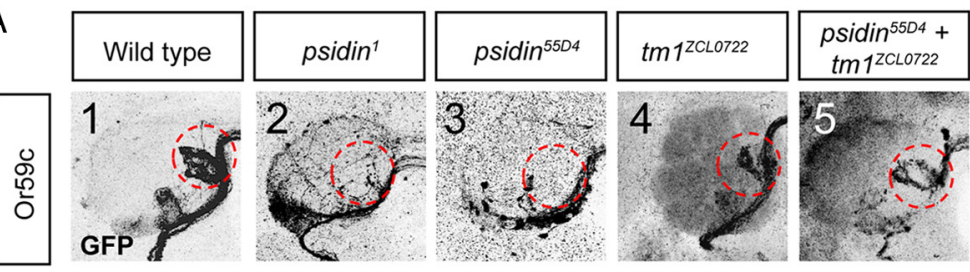

B
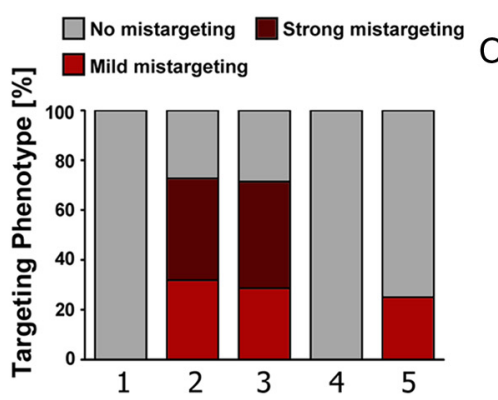

D
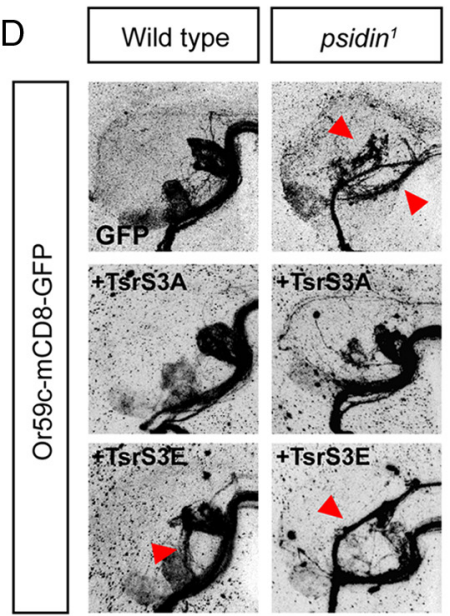

F
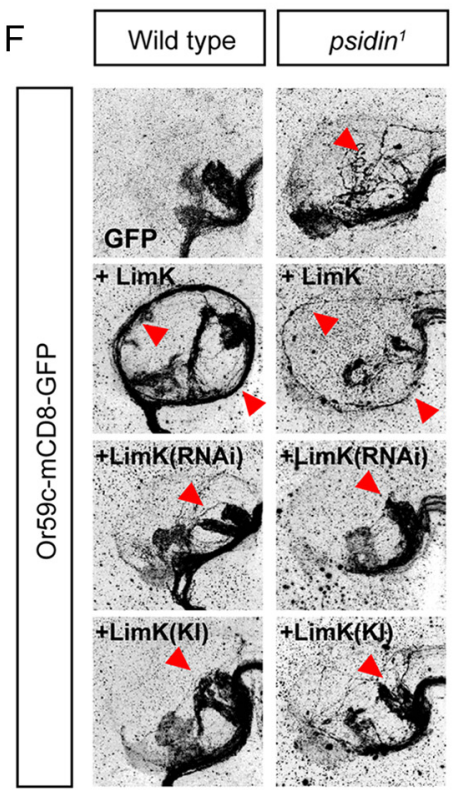

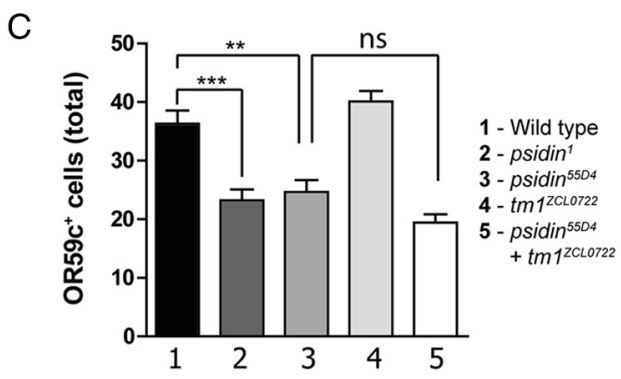

$\mathrm{E}$

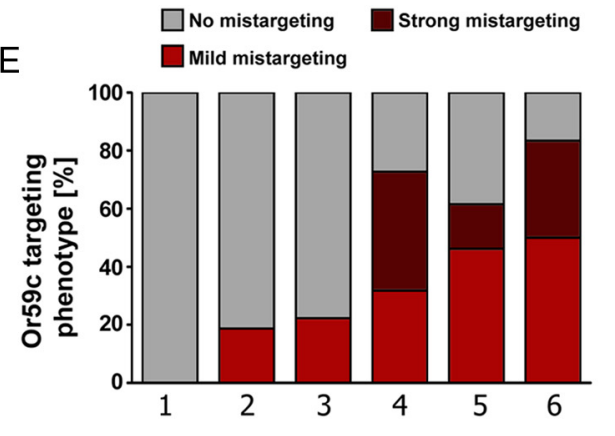

1 - Wild type

2 - Wild type $+\mathrm{Tsr}^{\mathrm{S} 3 \mathrm{~A}}$ (const. act.)

3 - Wild type $+\mathrm{Tsr}^{\mathrm{S} 3 \mathrm{E}}$ (inact.)

4 - psidin $^{1}$

5 - psidin $^{1}+\mathrm{Tsr}^{\mathrm{S3A}}$ (const. act.)

6 - psidin $^{1}+\mathrm{Tsr}^{\mathrm{S} 3 \mathrm{E}}$ (inact.)

G

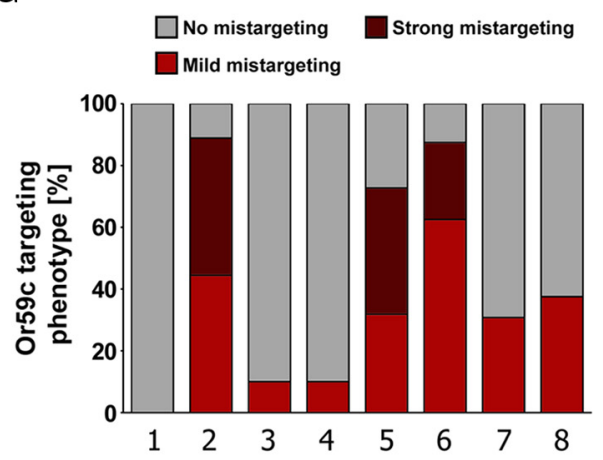

1 - Wild type

2 - Wild type + LimK (wildtype)

3 - Wild type + LimK (RNAi)

4 - Wild type + LimK (kinase inactive)

5 - psidin $^{1}$

6 - psidin + LimK (wildtype)

$7-p$ sidin $^{1}+$ LimK (RNAi)

8 - psidin ${ }^{1}+$ LimK (kinase inactive)

Figure 5. Psidin and Tropomyosin have opposing roles during axon targeting. A, Or59c neurons in a psidin ${ }^{7}$ and a psidin ${ }^{5504}$ mutant background showed a complete loss of innervation at their innate glomerulus (dashed circles). The tropomyosin mutant $t m 7^{\text {CLCO722 }}$ did not show any targeting defect (dashed circles). The targeting pattern of psidin single mutants was restored in psidin ${ }^{5504}$ plus $t m 7^{7^{Z C L O 722}}$ double mutants (dashed circles). $\boldsymbol{B}$, Single mutants of $t m 7^{\text {ZCLOT22 }}$ show no targeting defect, whereas psidin $^{7}(72 \%)$ and psidin ${ }^{5504}(71 \%)$ single mutants showed a pronounced overall targeting defect. The targeting defect of psidin ${ }^{5504}$ mutants was rescued in the double-mutant psidin ${ }^{5504}$ plus $t m 7^{7 C L 0722}(25 \%)$. C, The loss of Or59c neurons in the

Antibodies

The following primary antibodies were used: rabbit anti-GFP (1:1000; Clontech), mouse antiNC82 (1:20; DSHB), rat anti-HA (1:1000; Roche), and rabbit anti-myc (1:1000; Abcam). Secondary antibodies were used as follows: antimouse Cy5 (1:200; Dianova), anti-rabbit 488 (1: 200; Dianova), anti-rat HRP (1:1000; Jackson ImmunoResearch), anti-rabbit HRP (1:1000; Jackson ImmunoResearch), anti-Psidin (1:50; Kim et al., 2011), anti-Elav (1:50; Hybridoma Bank), rat anti-tubulin (1:1000; Abcam).

\section{Yeast experiments}

Saccharomyces cerevisiae strain YZ1143 (mdm20 deletion was kindly provided by Dr. F. S. Sherman, University of Rochester Medical Center, Rochester, NY) carrying a complementing plasmid, p[CEN URA3 MDM20-3HA], was used for the analysis. Integrative vector pRS405 (LEU2) carrying either a wild-type MDM20-myc or a mutant allele MDM $20{ }^{\mathrm{K} 304 \mathrm{E}}$-myc was digested by $\mathrm{XcmI}$ to facilitate integration into the yeast genome. Selected transformants were grown on 5-Fluoroorotic acid plates lacking leucine to remove the complementing plasmid. The presence of integrated allele was confirmed by PCR and subsequent sequencing. Temperature sensitivity was determined at $30^{\circ} \mathrm{C}$ and $37^{\circ} \mathrm{C}$. For actin staining, exponentially growing cells were fixed with formaldehyde for $10 \mathrm{~min}$, washed three times in PBS, and resuspended in Alexa-fluor-phalloidin (1:1000). After a $30 \mathrm{~min}$ incubation at room temperature, the cells were washed with PBS, mounted on slides in 70\% glycerol/PBS/0.05\% paraphenylenediamine, and immediately visualized.

\section{Results}

A mutation in the psidin gene affects olfactory neuron circuit formation

Using mosaic histological screening of $\sim 6000$ EMS mutants, we addressed the molecular mechanisms required for ORN axons to navigate to their correct targets within the AL. We used the eyFlp mosaic system, which induces mutant clones in the antenna and MP in addition to in the eye,

\footnotetext{
$\leftarrow$

psidin ${ }^{5504}$ background ( $n=25$ ) was not rescued in the double mutant $(n=20)$. The $t m 7^{\text {2CLO722 }}$ background did not show any significant reduction of $0 \mathrm{r} 59 \mathrm{c}$ neurons. $\boldsymbol{D}, \boldsymbol{E}$, Overexpression of constitutively active (const. act.) Cofilin partially rescues the psidin ${ }^{7}$-targeting phenotype ( $72 \%$ vs $58 \%$ ). Expression of inactive (inact.) Cofilin aggravates the phenotype (83\%). Expression of both cofilin variants leads to a mild phenotype in the wild-type background. $\boldsymbol{F}, \mathbf{G}$, Overexpression of LimK in a wild-type background leads to a strong targeting defect. Axons innervate along the outer rim of the AL (red arrowheads). Knockdown and overexpression of a kinase inactive form leads to a mild phenotype. The overall targeting phenotype in $p \operatorname{sidin}^{1}(72 \%)$ can be further aggravated by the overexpression of LimK (88\%). Knockdown or expression of DN LimK ( $72 \%$ vs $31 \%$ or $38 \%$ ) rescues the targeting phenotype, especially the strong targeting phenotype. ${ }^{*} p<0.05$; ${ }^{* *} p<$ $0.01 ;{ }^{* * *} p<0.001$, one-way ANOVA and Bonferroni's posttest. Error bars \pm SEM.
} 
but not in the central brain (Newsome et al., 2000). These clones usually resulted in $\sim 50 \%$ of all ORNs being homozygous for the respective mutant allele. Using specific reporter lines for distinct ORN classes (see Materials and Methods), we analyzed the effects that the mutations had on the synaptic targeting patterns of different ORN subclasses.

We identified a mutant IG978 displaying ORNs that failed to innervate their correct target glomeruli (Fig. $1 A$ ). Using deletion and SNP mapping, we mapped the mutant to the psidin locus (phagocyte signaling impaired; Fig. 1C). Psidin is the homolog of the yeast protein MDM20. MDM20 is the noncatalytic subunit of the $\mathrm{N}$-acetyltransferase complex NatB known in yeast to acetylate $\sim 60 \%$ or more of all cytosolic proteins on specific N-terminal amino acid residues (Polevoda and Sherman, 2003a; Singer and Shaw, 2003). Psidin was identified previously in Drosophila in two independent screens: as a lysosomal protein in blood cells (Brennan et al., 2007; Kim et al., 2011) and as a novel actinbinding protein essential for oocyte migration (Kim et al., 2011). To validate and understand the role of Psidin during olfactory circuit formation, we analyzed three different alleles in parallel: our newly established $p$ sidin $^{I G 978}$ allele (harboring the missense mutation E320K) and two expected null alleles, sidin $^{I}$ (Brennan et al., 2007) and $p$ sidin ${ }^{55 D 4}$ (Kim et al., 2011) (harboring a STOP codon at K441 and K471, respectively; Fig. 1C). These analyses revealed two phenotypes. First, psidin null alleles revealed an apparent reduction in axon numbers in several, but not all, ORN classes (Fig. 1A,E; Table 1). This phenotype was caused by a reduction in neuron numbers of these ORN classes, which was already visible at around $8 \mathrm{~h}$ after puparium formation (APF) in developing pupae (Fig. $1 E$ and data not shown), and therefore seemed to relate to a defect in early neurogenesis or cell survival (see below). Psidin ${ }^{I G 978}$ mutant clones showed a trend similar to psidin $^{l}$ mutants but no significant change in neuron numbers compared with controls (Fig. 1E).

Second, all three psidin mutant alleles revealed defects in glomerulus targeting, consistently affecting the same subclasses of ORNs (Fig. 1A,D; Table 1). The nature of these phenotypes was comparable regardless of whether ORN numbers were reduced ( $p \operatorname{sidin}^{1}$, psidin $^{55 D 4}$ ) or unaffected ( $p \operatorname{sidin}^{I G 978}$ ). Or47aexpressing ORNs target the dorsomedial glomerulus DM3 in control animals (Fig. 1A). In psidin mutants, they frequently innervated a ventromedial glomerulus (Fig. $1 A, D$ ). The majority of the ventromedial targeting ORNs (e.g., Or59c, Or42a, and Or92a) displayed strong mistargeting and ectopic synapse formation (Fig. $1 A, D$; Table 1). In contrast, centrally projecting ORNs like Or22a, Or47b, and Or71 a axons, which travel a short distance at a straight angle from the AL entry point, were hardly affected (Fig. 1A,D; Table 1).

Since the targeting tissue in the AL was not genetically manipulated by eyFlp, potential phenotypes were expected to affect the ORNs but not their target cells in the brain. We sought additional evidence that Psidin was required within the mistargeting ORN by using single cell and reverse MARCM analysis to visualize selectively wild-type axons in a mutant background. In these experiments, wild-type Or47a, 42a, and 59c ORNs within nonlabeled mutant ORNs projected normally, confirming that Psidin is required in a cell-autonomous manner in ORNs (Fig. $1 B)$. In addition, hs-Flp-mediated recombination to induce very small mutant ORN clones showed that single mutant axons mistargeted in psidin clones (Fig. $1 B$ ). To confirm the requirement of Psidin, we used the GAL4/UAS system to reexpress Psidin in ORNs homozygous for psidin (eyFLP clones; Fig. 2A). In these experiments, targeted expression of Psidin achieved successful rescue of ORN cell numbers and pathfinding phenotypes (Fig. 2A).

To understand the reason for the reduced number of ORNs, we expressed the antiapoptotic and caspase-inhibiting protein p35 (Davidson and Steller, 1998) in psidin $^{1}$ and psidin $^{\text {IG978 }} \mathrm{mu}$ tant and control clones. In these experiments, the cell number defect of sidin $^{1}$ mutant ORNs was fully rescued, but axonal targeting defects persisted (Fig. 2). These findings suggest that Psidin prevents apoptosis of ORNs during development. It also strongly supported the conclusion from our anatomical studies that the role of Psidin in axon navigation represents an independent function. Therefore, we conclude that Psidin-deficient defects in olfactory circuit formation are caused by two distinct cellular mechanisms.

\section{Psidin is expressed at the right stages in the developing olfactory organs}

We next analyzed the expression of psidin during different developmental stages using in situ hybridization and antibody staining (Fig. 3). Both methods suggested that Psidin is expressed ubiquitously in all cells, including the developing olfactory organs. Using in situ hybridization, psidin expression was detected at 5-10 h APF in the eye-antennal disc, the primordia of MP and antenna (Fig. 3A, B). This expression is consistent with a cell-autonomous role of Psidin in ORNs during neuronal differentiation, since ORNs arise at 6-20 h APF (Rodrigues and Hummel, 2008). At around $24 \mathrm{~h} \mathrm{APF}$, expression reached its maximum in both the $\mathrm{MP}$ and the antenna (Fig. $3 C, D$ ). At 30 and $45 \mathrm{~h}$, expression started to decline in the olfactory organs (Fig. $3 E-H$ ). This expression is coherent with a role in the axonal pathfinding of ORNs, known to start around $20 \mathrm{~h}$ APF and continue over a period of $\sim 30 \mathrm{~h}$ (Rodrigues and Hummel, 2008). We next asked whether Psidin protein was expressed in ORNs during development. ORs start to be expressed only after development of the olfactory system. Therefore, we used the pan-neuronal marker Elav to label all neurons. Analysis of protein expression using an anti-Psidin antibody showed that Psidin protein was expressed in neuronal marker Elav-positive cells likely to be developing ORNs, judging by location in the antennal disc (Fig. 3I). Thus, we conclude that Psidin is expressed during the relevant developmental times in ORNs both during neuron differentiation and during axonal targeting.

\section{Psidin promotes lamellipodia of neuronal growth cones}

Recent data showed that Psidin is a novel actin-binding protein required for the migration of Drosophila oocytes (Kim et al., 2011). To unravel how Psidin might contribute to the process of axonal navigation and whether similar mechanisms are required as for oocyte migration, we turned to cultures of embryo-derived primary Drosophila neurons that are ideally suited for the genetic dissection of cytoskeletal mechanisms underpinning axonal growth (Matusek et al., 2008; Sánchez-Soriano et al., 2010; Gonçalves-Pimentel et al., 2011). We found that psidin ${ }^{1}$, psidin $^{55 D 4}$, or $p_{\text {sidin }}{ }^{I G 978}$ mutant neurons displayed significantly smaller lamellipodia compared with wild-type controls (Fig. $4 A, B)$. These findings were consistent with potential roles of Psidin in actin regulation. Furthermore, neurites were significantly longer (Fig. $4 B$ ), similar to observations for other actinregulating proteins with known pathfinding defects in vivo (Sánchez-Soriano et al., 2010). HA-tagged Psidin partially colocalized with actin filaments in neurites and growth cones (Fig. 4C). These data were in agreement with the above-mentioned report that Psidin binds actin and regulates lamellipodia during 
A

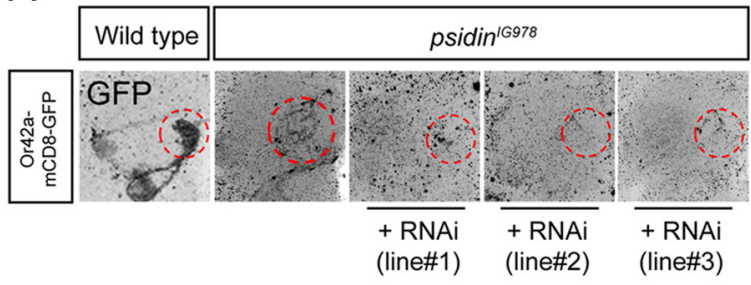

B

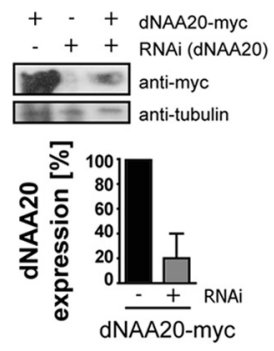

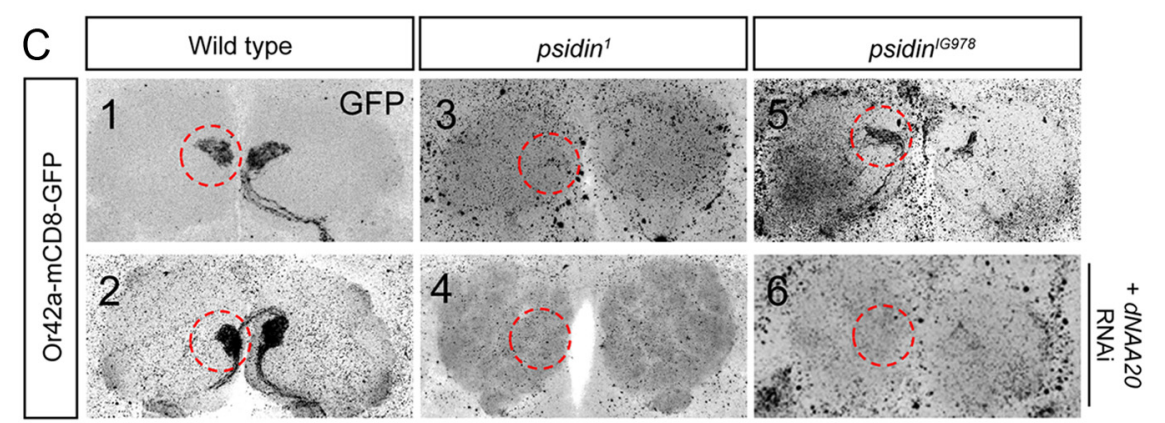

1 - Wild type

2 - Wild type + dNAA2O RNAi

3 - psidin $^{1}$

4 - $\operatorname{sidin}^{1}+d N A A 20$ RNAi

5 - psidin $^{\prime G 978}$

6 - sidin $^{\prime 6978}+$ dNAA20 RNAi

D

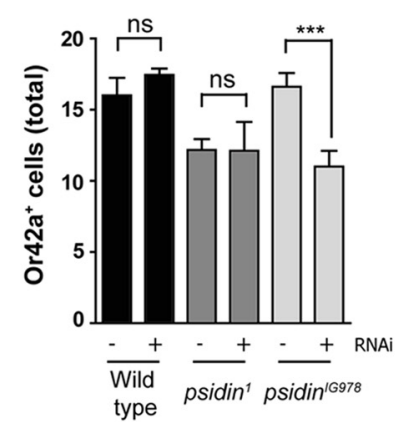

\section{$\mathrm{E}$}

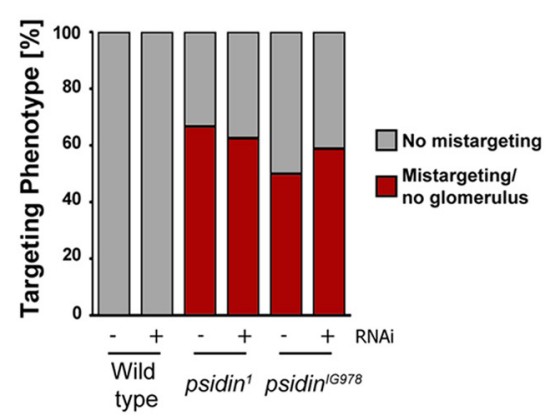

F

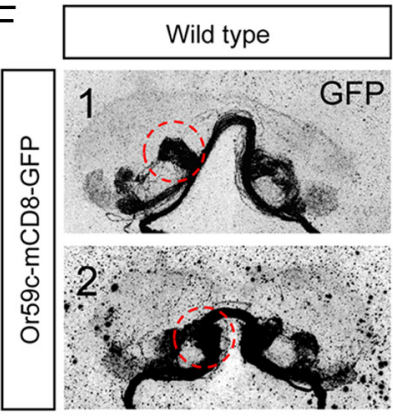

1 - Wild type

2 - Wild type + dNAA20 RNAi

3 - psidin 1

4 - psidin ${ }^{1}+$ dNAA20 RNAi

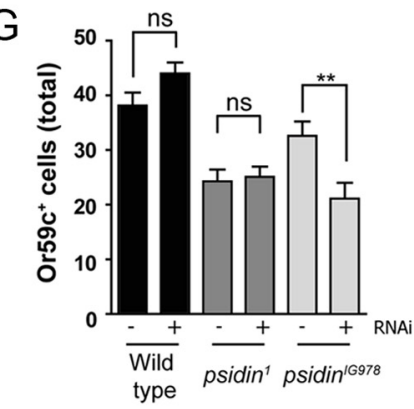

$\mathrm{H}$

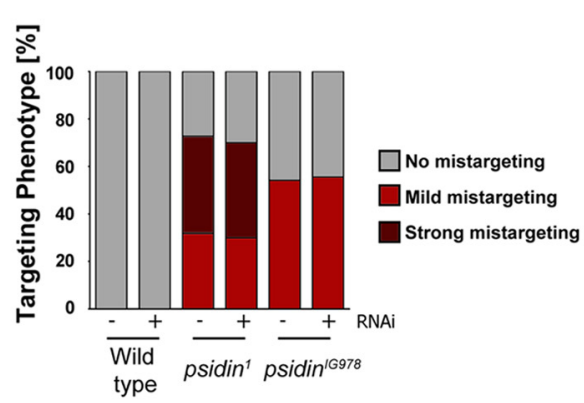

Figure 6. Psidin interacts with dNAA20 in vivo $\boldsymbol{A}$, The effect of dNAA20 on 0 RN targeting was assessed using RNAi. Act-GAL4 was used to drive the expression of RNAi in eyFlp clones. Three different RNAi lines were used to knock down dNAA20 in 0r42a

border cell migration in Drosophila oocytes (Kim et al., 2011). In that study, Psidin was suggested to antagonize the function of the actin-stabilizing protein Tropomyosin 1 ( $\mathrm{Tm} 1$ ), thus maintaining actin networks in a dynamic state. To test whether a similar mechanism might apply to Drosophila neurons, we analyzed $t m 1^{\text {ZCLO722 }}$ mutant neurons and found no detectable phenotypes (Fig. $4 A, B$ ). However, when the $t m 1^{\text {ZCLO722 }}$ mutation was combined with the psidin ${ }^{55 D 4}$ null mutant, the Psidin-deficient axon length and lamellipodial phenotypes were reverted to wild-type levels (Fig. 4A,B). These data were consistent with observations in non-neuronal cells and suggested that the psidin mutant phenotype is caused by unrestrained Tm1 activity.

We asked whether the point mutation in Psidin ${ }^{\text {IG978 }}$ that causes defects in lamellipodia size and axon targeting, but not in cell survival, would interfere with actin binding and thereby explain the observed phenotype. Using an in vitro actin-binding assay, we found that Psidin ${ }^{\text {IG978 }}$ bound actin at similar levels as wild-type Psidin (Fig. 4D). Thus, unidentified mechanisms in addition to actin binding must be involved in Psidin's regulation of lamellipodia size and actin dynamics.

neurons. All three lines had the same effect. $\boldsymbol{B}$, Quantification of dNAA20 knockdown. $\$ 2$ cells were transfected with dNAA20-myc and RNAi against dNAA20 (line\#2). After coexpression of the RNAi against dNAA20, protein levels were reduced by $\sim 70-80 \%$ compared with expression of dNAA20-myc alone. C, Knockdown using RNAi (line\#2) of dNAA20 in the psidin ${ }^{16978}$ mutant background showed a loss of glomerular innervation of Or42a neurons similar to the $p$ sidin $^{1}$ mutant background. D, Quantification of the total 0 r42a cell population: knockdown leads to reduction of Or42a neurons comparable to levels of $p s^{2} i^{1}{ }^{1}$ mutants. No further reduction was observed in the $p$ sidin $^{1}$ mutant after dNAA20 knockdown. $E$, Quantification of the Or42a mistargeting phenotype expression of RNAi had no effect on the targeting phenotype. $\boldsymbol{F}$, The effect of dNAA20 on Or59c targeting. The targeted glomerulus of 0 r 59 c neurons appears not to be innervated in psidin ${ }^{7}$ mutant flies (dashed circles). Axons display an overall random innervation pattern across the AL (arrowhead). The targeting phenotype does not change after expression of RNAi. Comparably, the expression of RNAi has no effect on psi$\operatorname{din}^{16978}$ mutant neurons. G, Quantification of the total Or59c cell population: knockdown using RNAi (line\#2) of dNAA20 in the psidin ${ }^{16978}$ mutant background leads to a significant reduction of 0 r 59 c neurons, to levels comparable to $p \operatorname{sidin}^{7}$ mutants. No further reduction of the neuron number was observed after knockdown in psidin ${ }^{7}$ mutants. The knockdown of dNAA20 had no effect on wild-type cells. $\boldsymbol{H}$, Quantification of the targeting phenotype of Or59c axons expression of RNAi in psidin ${ }^{7}$ and $p \operatorname{sidin}^{16978}$ had no effect on targeting. ${ }^{*} p<0.05 ;{ }^{* *} p<0.01 ;{ }^{* * *} p<0.001$, one-way ANOVA and Bonferroni's posttest. Error bars \pm SEM. 


\section{Roles of Psidin in maintaining dynamic actin networks can explain its in vivo roles}

We next tested whether the role of Psidin in restraining Tm1 activity in growth cones is of relevance for the axon-targeting phenotype of ORNs in vivo (Fig. 5). For this, we analyzed the two independent loss-of-function mutant alleles, $t m 1^{\text {ZCLO722 }}$ (Morin et al., 2001; Kim et al., 2011) and $t m 1^{\text {sufflw)4 }}$ (Vereshchagina et al., 2004), using eyFlp analysis. Consistent with our findings in growth cones, both alleles displayed no defects, neither in axonal projection patterns nor in the number of Or59c-expressing ORNs (Fig. $5 A-C$ ). Also consistent with our findings in primary neuron cultures, ORN homozygous mutants for both psidin ${ }^{5504}$ and $t m 1^{Z C L O 722}$ showed a marked reduction in the percentage of brains that display Or59c axonal targeting defects compared with psidin ${ }^{55 D 4}$ mutants alone (Fig. $5 A, B ; 70 \%$ vs $20 \%$ ). However, the psidin $^{55 D 4}, \operatorname{tm}^{\text {ZCLO722 }}$ double-mutant condition did not rescue the reduction in the number of Or59c-expressing ORNs typical of the $\operatorname{sidin}^{55 D 4}$ or $p$ sidin ${ }^{1}$ single-mutant backgrounds (Fig. 5C). These data suggest that Psidin functions as a regulator of actin dynamics in conjunction with $\mathrm{Tm} 1$ during axon targeting (see also Kim et al., 2011). In contrast, it implicates a Tm1-independent mechanism of Psidin during neuronal survival.

$\mathrm{Tm} 1$ acts primarily as a stabilizer of F-actin (Bugyi and Carlier, 2010). Another factor reported to compete with Tm1 is ADF/ cofilin (Kuhn and Bamburg, 2008). Therefore, we tested whether Drosophila cofilin (called Twinstar/Tsr) has an impact on Psidin function. For this, we overexpressed a constitutive active (S3A) and a constitutive inactive version (S3E) of Tsr in wild-type and psidin $^{1}$ mutant clones. Overexpression of either of the two Tsr isoforms in wild-type clones resulted in mild mistargeting of Or59c ORN axons in $\sim 20 \%$ of the brains ( $\mathrm{Tsr}^{\mathrm{S3A}}, 20 \%$; $\mathrm{Tsr}^{\mathrm{S}}{ }^{3 \mathrm{E}}$, 23\%; Fig. $5 D, E$ ). However, constitutive active $\mathrm{Tsr}^{\mathrm{S3A}}$ overexpressed in psidin ${ }^{1}$ mutant Or59c ORNs reduced their mistargeting phenotype by $14 \%$ compared with $p \operatorname{sidin}^{1}$ alone, consistent with a compensatory role of cofilin through competing with $\mathrm{Tm} 1$ for actin. Vice versa, inactive $\mathrm{Tsr}^{\mathrm{S}}{ }^{\mathrm{E}}$ aggravated the psidin $^{l}$ phenotype ( $8 \%$ increase; Fig. $5 D, E)$, likewise consistent with the above idea.

Cofilin function in F-actin disassembly is negatively regulated by phosphorylation through LimK, which was previously implicated in ORN targeting (Ang et al., 2006). We found that expression of wild-type LimK in wild-type ORNs caused $88 \%$ of Or59c ORNs to mistarget (Fig. $5 F, G$ ). Expression of LimK RNAi or of a kinase-inactive LimK affected the targeting of only $10 \%$ of ORNs (Fig. $5 F, G$ ). Expression of wild-type LimK in psidin $^{l}$ mutant ORNs resulted in a comparable degree of targeting defects as the same experiment caused in wild-type neurons ( $87 \%)$. This value was slightly higher than that of sidin $^{1}$ mutant ORNs without targeted LimK expression (Fig. $5 F, G$ ). Consistent with the idea that Psidin promotes actin dynamics and instability, overexpression of LimK RNAi or kinase-inactive LimK (i.e., those conditions that promote cofilin activity) significantly rescued the psidin $^{l}$ mutant axonal targeting phenotype (Fig. $5 F, G$ ). The results confirm that Psidin's function as an actin regulatory protein, originally discovered in non-neuronal cells, can be applied in the context of neuronal guidance. Furthermore, we conclude that Psidin's role in actin dynamics is required for growth cone targeting but is not involved during ORN survival.

Neuron number but not axon targeting depend on Psidin as a subunit of the $\mathrm{N}$-acetyl transferase B complex

We next addressed potential molecular mechanisms of Psidin function during the regulation of neuron number. Psidin is the homolog of yeast MDM20, the noncatalytic subunit of the evolutionarily well conserved $\mathrm{Nat} \mathrm{B}$ complex required for $\mathrm{N}$-terminal protein acetylation (Polevoda and Sherman, 2003a). In budding yeast, nat $B$ subunit mutants show defects including mitochondrial inheritance, budding, and cell division (Polevoda and Sherman, 2003b; Singer and Shaw, 2003). A role in cell growth was also found using human cell culture (Starheim et al., 2008). To test whether Psidin's role in ORN survival reflects a function as a constituent of the NatB complex, we investigated the Drosophila gene CG14222 (referred to as dNAA20 in the following) encoding the predicted catalytic subunit of NatB. Because of the lack of dNAA20 mutants or suitable deletions, we used eyFlp in combination with a strong driver (act-GAL4) and expressed three independent RNAi constructs selectively in ORNs (Fig. 6A). All constructs showed comparable phenotypes (Fig. 6A). The RNAi used for the experiments achieves strong knockdown of dNAA20 levels (Fig. 6B), but its expression in ORNs of wild-type flies was insufficient to cause neuron loss or axon-targeting phenotypes in Or59c or Or42a neurons (Fig. 6C, E, F,H). Expression of $d N A A 20$ RNAi in clones mutant for the hypomorphic allele psidin ${ }^{I G 978,}$ however, resulted in a significant decrease in ORN numbers, reminiscent of phenotypes observed in psidin ${ }^{l}$ mutant clones (Fig. $6 D, G$ ). This suggests that $p^{\text {sidin }}{ }^{I G 978}$ provides a sensitized background for knockdown of $d N A A 20$, and it is consistent with the trend in neuron number reduction in hypomorphic mutant clones (see Fig. 1). Or59c and Or42a neuron numbers in psidin ${ }^{1}$ null mutants were not further decreased by $d N A A 20$ RNAi expression, suggesting that Psidin's role in neuron survival is $\mathrm{NatB}$ dependent (Fig. $6 D, G$ ). In contrast to the reduction in cell number, Or59c and Or42a ORNs did not show any enhancement in mistargeting or ectopic synapse formation when $d N A A 20 \mathrm{RNAi}$ was expressed in psidin ${ }^{I G 978}$ or psidin ${ }^{1}$ mutants (Fig. 6E,H). These results indicate that $d N A A 20$ is dispensable for ORN targeting but is required for the survival of the correct number of ORNs in a Psidin-dependent manner.

\section{The central domain of Psidin binds the catalytic NatB subunit dNAA20}

We next tested whether dNAA20 and Psidin physically interact. To this aim, we used coimmunoprecipitation assays of HAtagged Psidin with myc-tagged dNAA20, both coexpressed in S2 cells (Fig. 7A). Pulldown of wild-type Psidin with anti-HA antibody resulted in significant amounts of coimmunoprecipitated dNAA20 (Fig. 7A). These data show that Psidin and dNAA20 form a complex, as has similarly been reported for their yeast and human homologs (Singer and Shaw, 2003; Starheim et al., 2008). Comparable coimmunoprecipitation was achieved with Psi$\operatorname{din}^{\text {IG978 }}$, suggesting that this mutation does not interfere with NatB complex formation (Fig. 7A). The same physical interaction was observed in the reverse experiment using anti-mycmediated pulldown (data not shown).

Next we sought to map the interaction domain of Psidin with dNAA20 within the Psidin protein. In addition to the Tpr- and coiled-coil domains, a so-called putative NatB interaction domain is predicted by some software programs (Fig. $1 B$ and SMART Heidelberg). We deleted this domain entirely ( $\triangle$ NatBfull) or in parts ( $\triangle \mathrm{NatB} 1-3)$ from Psidin and used it to test coimmunoprecipitation of dNAA20 from S2 cell lysates (Fig. 7C). The deletion of the entire domain resulted in complete loss of coimmunoprecipitated dNAA20 (Fig. 7C). Subdeletions of the $\mathrm{N}$-terminal, middle, or C-terminal parts of the domain resulted in partial loss of interaction (Fig. 7C,D). The strongest interaction was maintained with a deletion of the region where Psidin ${ }^{\text {IG978 }}$ 
A

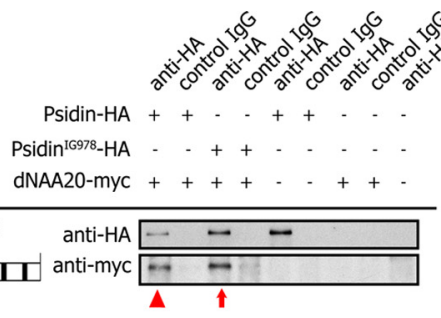

Input:

Psidin-HA +

Psidin ${ }^{\mathrm{IG} 978}-\mathrm{HA} \cdot+\cdots$

dNAA20-myC $++\cdot+$

anti-HA $-\mathrm{Cr}$

anti-myc

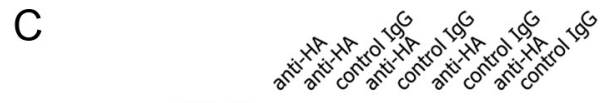

Psidin-HA

Psidin Deletion-HA

dNAA20-myc +

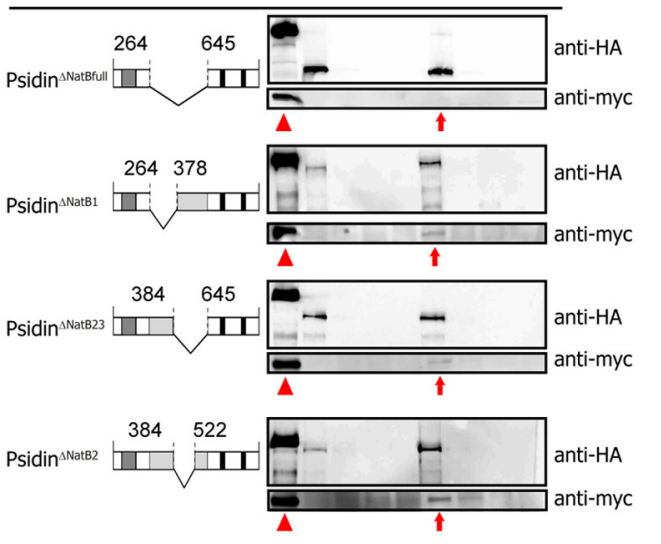

Psidin $^{\text {wt }}+\cdot \cdot \cdot \cdot \cdot \cdot \cdot \cdot \cdot \cdot$

Input:

dNAA20 + + - + + + + +

Psidin $^{\triangle \mathrm{NatB1}}-++\cdots+-\cdot \cdot$

Psidin $^{\triangle \mathrm{NatB2}}-\cdot \cdot++\cdot \cdot \cdot \cdot$

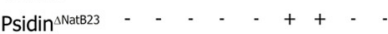

Psidin ${ }^{\Delta \text { Natffull - }}$
B

\begin{tabular}{|ll|}
\hline KLELIRRL & Homo sapiens \\
KLELIRRL & Mus musculus \\
HLELIRRL & Danio rerio \\
RLELHQRM & Drosophila (consensus) \\
RLKLDIMY & Saccharomyces cerevisiae \\
IGKLTKQI & Caenorhabditis elegans \\
\hline
\end{tabular}

\section{D}

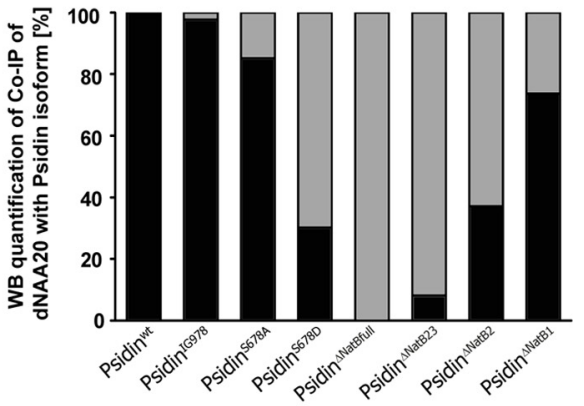

$\mathrm{F}$

\begin{tabular}{|ll|}
\hline IRSLMLRL & Homo sapiens \\
IRSLMLRL & Mus musculus \\
IRSLTLRL & Danio rerio \\
VRSLMLRL & Drosophila (consensus) \\
LTLIRELI & Saccharomyces cerevisiae \\
IRSTLCRA & Caenorhabditis elegans \\
\hline
\end{tabular}

anti-HA

$=-\infty-\infty$

anti-myc
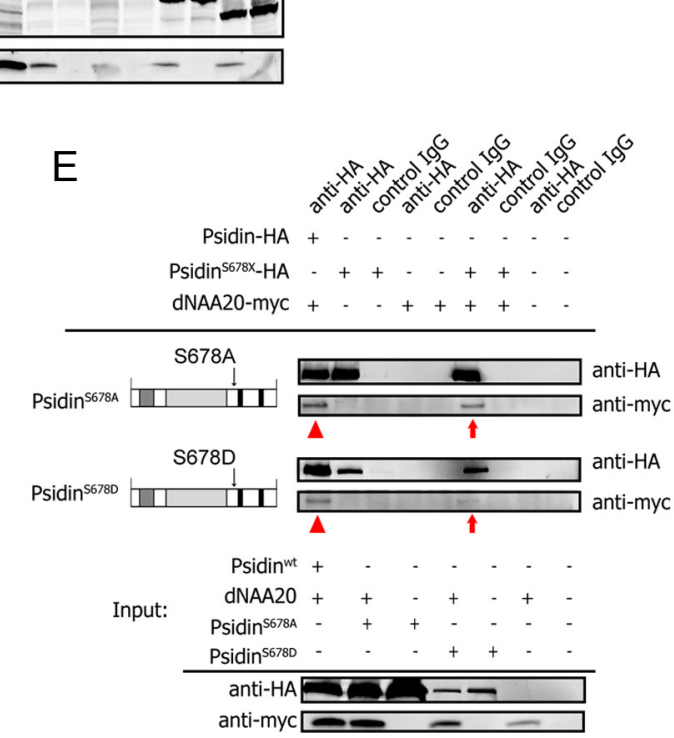

Figure 7. Psidin interacts with dNAA20 in vitro A, S2 cells were cotransfected with Psidin-HA/Psidin ${ }^{16978}$-HA and dNAA20-myc. dNAA20 was coimmunoprecipitated with wild-type Psidin (arrowhead) and Psidin ${ }^{16978}$ (arrow) isoforms in S2 cells at comparable levels. The protein Psidin ${ }^{16978}$ carries the point mutation found in psidin ${ }^{16978}$ mutants. B, The position E320 is highly conserved in higher organisms having a glutamate at this position. Interestingly, the yeast wild type resembles exactly the mutation found in the psidin ${ }^{16978}$ allele. C, Representative examples of coimmunoprecipitated dNAA20-myc with various Psidin deletions. The top blot shows the different Psidin deletions (anti-HA blot). The bottom blot displays the amount of (Figure legend continues.) 
carries the point mutation E320K ( $\Delta$ NatB 264-378; Fig. 7C). We quantified the level of bound dNAA20 to the Psidin deletions relative to the binding of dNAA20 to the wild-type Psidin protein (Fig. $7 D$ ). We conclude that the putative NatB interaction domain is indeed required for the interaction of dNAA2 0 and Psidin.

\section{Phosphorylation of a conserved serine in Psidin prevents NatB complex formation and interferes with Psidin's function during ORN survival}

Our next experiments were designed to more precisely distinguish the dNatB-dependent and -independent roles of Psidin in vivo. Deleting the entire or parts of the NatB interaction domain appeared very crude and not well suited for in vivo experiments. Therefore, we addressed potential mechanisms for regulated interaction between dNAA20 and Psidin. Previously, a conserved serine ( $\mathrm{S} 678$ in fly; Fig. 7F) has been shown to be phosphorylated in human MDM20 using mass spec analysis (Trost et al., 2009). As this serine is located just downstream to the NatB interaction domain, we generated mutants that would either mimic phosphorylation (S678D) or make phosphorylation impossible at this location (S678A). The nonphosphorylatable Psidin mutant S687A coimmunoprecipitated dNAA20 at similar levels to Psi$\operatorname{din}^{\text {IG978 }}$ and wild-type Psidin (Fig. $7 D, E$ ). In contrast, the phosphomimetic mutant S678D pulled down significantly lower amounts of dNAA20 compared with wild-type Psidin and Psi-

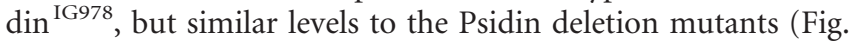
$7 D, E$, Psidin ${ }^{\mathrm{S} 678 \mathrm{D}}$ vs Psidin $\left.{ }^{\Delta \mathrm{NatB} 2}\right)$. Hence, this amino acid mutation provides an excellent opportunity to ask whether binding to dNAA20 is required for both of the in vivo functions we observed for Psidin. We generated transgenic flies and reexpressed three different point mutations within the Psidin protein in $p s i$ din mutant clones (Fig. $8 A-C$ ). First, we expressed Psidin ${ }^{\text {IG978 in }}$ psidin $^{1}$ and wild-type clones. UAS-psidin ${ }^{I G 978}$ fully rescued the cell-loss phenotype in psidin ${ }^{1}$ clones (Fig. 8 B). In contrast, the axon-targeting defect remained and was rescued only to the level of $\operatorname{sidin}^{I G 978}$ mutant clones (Fig. 8C). This result confirms that the single-point mutation in Psidin ${ }^{\text {IG978 }}$ is sufficient to interfere with axon targeting without affecting ORN numbers. Next, we expressed psidin ${ }^{\text {S678A }}$ and psidin ${ }^{\text {S678D }}$ in mutant and control clones. While expression of Psidin ${ }^{\text {S678A }}$ rescued both the cell-loss and axon-targeting phenotypes, expression of Psidin ${ }^{\text {S678D }}$ selectively rescued the axon-targeting phenotype but showed no

\footnotetext{
(Figure legend continued.) dNAA20 that was pulled down together with the Psidin variants (anti-myc blot). As a reference, wild-type Psidin pulldown of dNAA20 was used (arrowhead, left lane) to compare the pulldown of dNAA20 with Psidin deletions (arrow, right lane). Deletion of

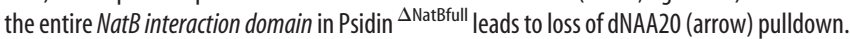
Smaller deletions of this domain (Psidin ${ }^{\Delta \text { NatB23 }}$, Psidin ${ }^{\Delta \text { NatB2 }}$ ) showed a reduced pulldown of dNAA20 (arrow). Only Psidin ${ }^{\triangle N a t B 1}$ was able to pull down dNAA20 (arrow) at levels close to wild-type Psidin (arrowhead). D, Quantification of the Western blots showing the normalized pulldown of dNAA20 with different Psidin isoforms. Psidin ${ }^{16978}$, Psidin ${ }^{5678 A}$, and Psidin ${ }^{\Delta N a t B 1}$ pulled down dNAA20 at wild-type levels. In contrast, Psidin ${ }^{56780}$, Psidin ${ }^{\Delta N a t B f u l l}$, Psidin ${ }^{\triangle N a t B 23}$, and Psidin ${ }^{\Delta N a t B 2}$ show a reduced or no pulldown of dNAA20, respectively. $\boldsymbol{E}$, Representative examples of coimmunoprecipitated dNAA20-myc with Psidin phosphomutants. The top blot shows the Psidin phosphomutant (anti-HA blot). The bottom blot displays the amount of dNAA20 that was pulled down together with the phosphomutant (anti-myc blot). As a reference, wild-type Psidin pulldown of dNAA20 was used (arrowhead, left lane) to compare the pulldown of dNAA20 with Psidin deletions (arrow, right lane). Psidin ${ }^{5678 \mathrm{~A}}$ was able to pull down dNAA20 (arrow) at levels similar to wild-type Psidin (arrowhead). In contrast, Psidin ${ }^{\text {5678D }}$ showed a reduced pulldown of dNAA20 (30\% of wild-type Psidin). $\boldsymbol{F}$, The serine residue at the position 678 in Drosophila is highly conserved among other organisms. Again, in yeast this residue seems not to be conserved.
}

ability to prevent cell loss (Fig. $8 A-C$ ). Given that Psidin ${ }^{\text {S678D }}$ binds dNAA20 significantly less, these data provide additional strong evidence for a dNAA20-dependent role of Psidin during neuron survival.

\section{Discussion}

The formation of a neuronal network depends on the proper development and targeting of neurons within the network. Here, we show that Psidin, in a similar manner to its recently described role in oocyte migration, acts as a constituent part of the actin machinery in the context of neuronal circuit formation in vivo. Furthermore, it functionally links to known actin regulators, in particular LimK and nonmuscle Tropomyosin. In addition, we define a novel role of Psidin in cell survival of neurons during development. Our data strongly suggest that Psidin uses two modes of activity. First, it prevents apoptosis of developing olfactory neurons as a noncatalytic subunit of the NatB complex. Second, it regulates axon targeting via its interaction with cytoskeleton regulators. Regarding the first role as part of the NatB complex, our results prove an evolutionarily conserved role of Psidin that has been predicted so far merely on the basis of in silico comparisons (see below) (Brennan et al., 2007).

For its second role, our findings essentially support the idea that cell migration and axonal growth share important properties (Lowery and Van Vactor, 2009). Axon guidance is coordinated through signaling processes downstream of extracellular biochemical cues and mechanical properties of the growth cone environment. The key effectors of these signaling processes are the actin- and microtubule-associating proteins, which determine the cytoskeletal dynamics underpinning any morphogenetic changes that growth cones undergo (Dent and Gertler, 2003; Lowery and Van Vactor, 2009). We show that loss of Psidin results in smaller growth cones with significantly reduced lamellipodia compared with wild-type controls. The aberrant growth cone morphology is completely rescued by the additional removal of Tm1, suggesting that Psidin promotes actin dynamics of growth cones by restraining Tm1 activity. Similar genetic relationships between Psidin, cofilin, and Tm1, as we found in neurons, were recently demonstrated in the context of oocyte migration (Kim et al., 2011).

We present a point mutation in Psidin (E320K) that selectively affects lamellipodia morphology, and hence axon targeting, but has no significant impact on neuronal cell numbers. Kim et al. (2011) demonstrated previously that Psidin physically binds actin in vitro. We reproduced this result and hypothesized that the E320K point mutation in Psidin ${ }^{\text {IG978 }}$ might interfere with Psidin's actin-binding ability, thus explaining the loss of function of this allele during axonal growth. However, Psidin ${ }^{\text {IG978 }}$ maintained its actin affinity, suggesting that actin interaction might be required for Psidin function in growth cones but that the key mechanism underlying this role is still to be uncovered. Differential screens for proteins interacting with Psidin in the region around position E320 might clarify this point. For example, Psidin might bind Tm1 directly, and this is not unlikely when considering links between the NatB complex and Tropomyosin in yeast (Singer and Shaw, 2003).

Not all ORN axon types require Psidin equally. While neurons that target dorsal and ventral glomeruli are strongly affected, axons that innervate central glomeruli of the AL show no or only mild mistargeting phenotypes. At first sight, this contradicts the highly penetrant phenotypes in embryonic primary neurons. Given that these primary neurons represent a broad range of different neuron types, we suggest that Psidin is part of the house- 

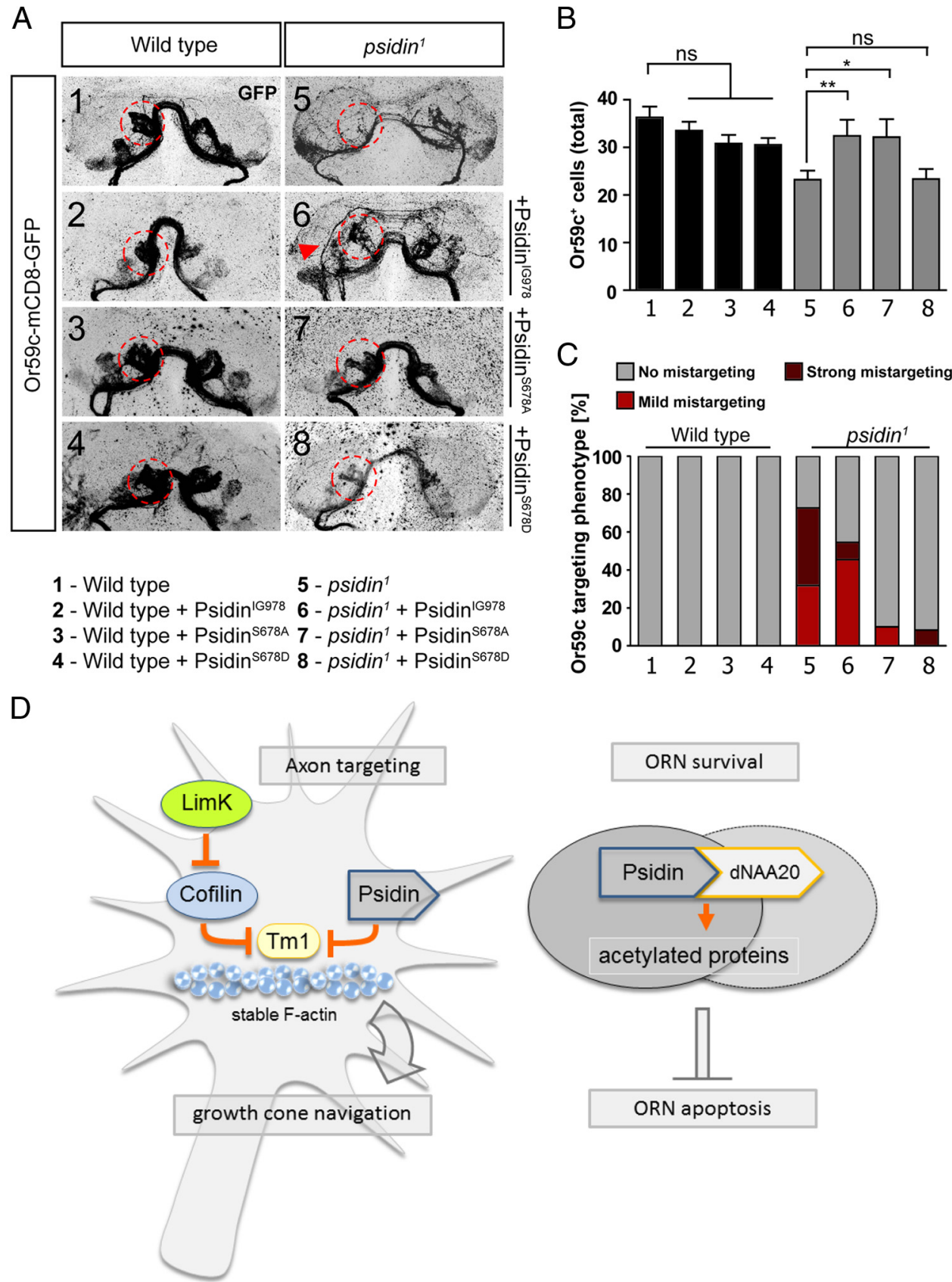

Figure 8. Conserved phosphorylated serine 678 prevents binding of dNAA20 to Psidin. A, Psidin isoforms were expressed in eyFlp clones driven by act-GAL4. Expression of Psidin ${ }^{16978}$ (carrying

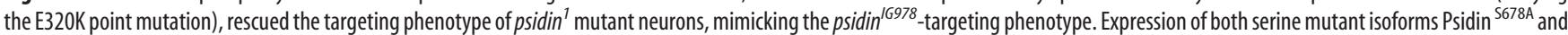
Psidin ${ }^{56780}$ rescued the targeting phenotype to wild-type levels. B, Quantification of the Or59c cell population: expression of Psidin ${ }^{16978}$ and Psidin $^{\text {S678A }}$ rescues the cell loss in the psidin ${ }^{7}$ background. Expression of the phosphomimetic Psidin ${ }^{56780}$ fails to rescue the cell loss. C, Quantification of the targeting phenotype. Expression of Psidin ${ }^{16978}$ in the psidin ${ }^{7}$ mutant background rescues targeting to $p s i d i{ }^{16978}$ levels. Expression of both serine mutants phosphomutants rescue the targeting to wild-type levels. D, Model of Psidin's two functions during axon targeting and ORN survival. During axon guidance of olfactory neurons, Psidin function as an actin-binding protein, preventing the formation of long and stable filaments in growth cones. It acts as an antagonist to the actin filament stabilizer Tropomyosin. LimK, possibly via inactivation of cofilin, allows actin filament stabilization, and inactivation of LimK rescues Psidin's requirement in targeting. For ORN survival, Psidin is required as a noncatalytic subunit of the NatB complex, in which it interacts with the catalytic subunit dNAA20 to regulate protein acetylation (e.g., during cell cycle). ${ }^{*} p<0.05$; ${ }^{* *} p<0.01$; ${ }^{* * *} p<0.001$, one-way ANOVA and Bonferroni's post-test. Error bars indicate \pm SEM.

keeping machinery of growth cones. We can think of two explanations. First, as suggested by the genetic interactions of Psidin not only with Tm1 but also with cofilin, different signaling pathways operating in distinct ORNs are likely to orchestrate their actin networks through different molecular routes, part of which appear redundant. This would mean that the degree of functional Psidin contributions is neuron and/or context specific. Second, even if Psidin was promoting flexible F-actin structures equally in all ORNs, the requirement for this lamellipodial property could be context dependent, consistent with the idea that intact F-actin networks in vivo are not required for axon extension per se, but for axon navigation (Sánchez-Soriano et al., 2010). For example, neurons using the dorsolateral and ventromedial targeting routes or traveling further distances are likely to be more dependent on dynamic actin networks and therefore more affected by the loss of Psidin than axons that do not turn or meet and adhere to their 
A
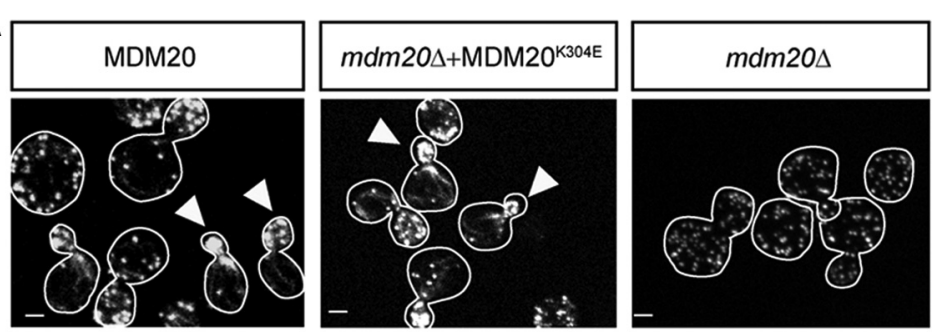

$\mathrm{B}$
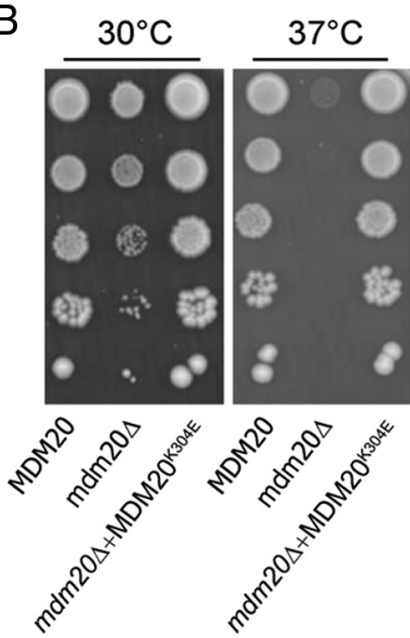

$\mathrm{C}$

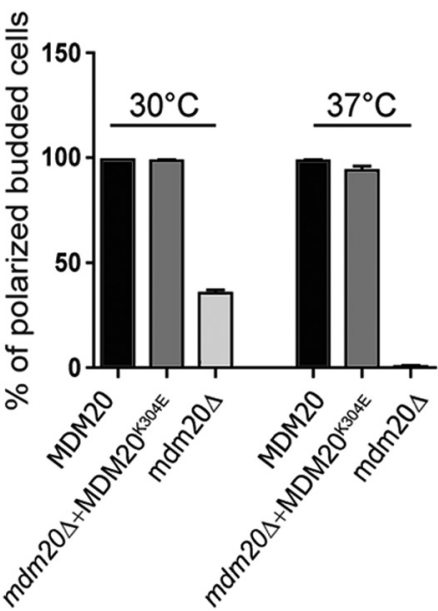

Figure 9. "Fly-like" yeast MDM20 ${ }^{\mathrm{K} 304 \mathrm{E}}$ rescues wild-type MDM20 function during cell division. A, Mimicking a fly-like MDM20 by replacing lysine with glutamate did not change the functionality of the yeast MDM20 protein compared with wild type. Expression of MDM20 ${ }^{\mathrm{K} 304 \mathrm{E}}$ in an $m d m 20 \Delta$ strain rescues the actin cable formation during cell division. $\boldsymbol{B}$, Expression of MDM20 ${ }^{\mathrm{K} 304 \mathrm{E}}$ can fully rescue the growth defect of $m d m 20 \Delta$ strain. $C$, The percentage of polarized yeast cells is not affected in MDM20 ${ }^{\mathrm{K} 304 \mathrm{E}}$-expressing cells under different growth conditions.

targets early on (centrally projecting ORNs). Judging from the distribution of the affected glomeruli and the fact that embryonic neurons in culture were equally affected by Psidin deficiency, we propose that the latter explanation is the more likely to be the correct one. Essentially, depending on the neuron's task in targeting to the correct glomerulus, the lack of Psidin and its effect on actin dynamics can be compensated for or turn into a visible phenotype. These data might provide additional evidence for the pivotal role of actin and cytoskeleton dynamics as an intrinsic property of a neuron. They also suggest that neurons with more challenging targeting routes require more complex guidance cues and downstream integrating signaling mechanisms at the level of the cytoskeleton.

Our data indicate that Psidin uses an evolutionarily conserved pathway via its function as a NatB complex subunit and interactor of dNAA20 to ensure ORN survival, likely by inhibiting their apoptosis (Polevoda and Sherman, 2003b; Starheim et al., 2008). However, the interaction domain within MDM20 or Psidin had only been predicted so far. We provide the first experimental evidence that the predicted domain within Psidin is indeed required for the interaction of Psidin and dNAA20. In addition, we identify a conserved serine just downstream of the interaction domain as a putative tool to regulate $\mathrm{NatB}$ complex formation in a phosphorylation-dependent manner. Given that this amino acid was found to be phosphorylated in human MDM20, it seems likely that this mechanism applies to humans as well. Our findings suggest that phosphorylation at this amino acid potently interferes with binding between Psidin and dNAA20. Furthermore, the failure of the phosphomimetic mutant to rescue the psidin cell-loss phenotype while rescuing the targeting defect strongly suggests that Psidin is required in two modes: nonphosphorylated as part of the NatB complex to regulate survival of neurons and phosphorylation independent as a regulator of actin dynamics in processes like cell migration and axon targeting. Interestingly, this serine is not conserved in yeast MDM20, indicating that this regulation was not present yet in yeast.

In contrast to psidin ${ }^{S 678 D}$, the psi$\operatorname{din}^{I G 978}$ mutation selectively impacts on axon targeting but not on neuron survival. Nevertheless, in addition to the targeting phenotype, the $\operatorname{psidin}^{I G 978}$ allele provides a sensitized background for knockdown of dNAA20. Although this functional interaction demonstrates a Psidin-dependent requirement of dNAA20, it also suggests that the Psidin ${ }^{\text {IG978 }}$ mutation affects a less critical and likely dNAA20-related function of Psidin during neuronal survival. Yet, neither protein stability or expression levels nor protein localization appear affected in psidin ${ }^{I G 978}$ mutants (data no shown). The fact that yeast wild-type MDM20 resembles the Psidin ${ }^{\text {IG978 }}$ mutant at this position further indicates that a glutamate or aspartate at this site is not essential for NatB function. A single amino acid mutation that we introduced into the yeast MDM20 to render it into a higher eukaryote-like version (K304E) did not interfere with MDM20's function during cell division and budding (Fig. $9 A, B$ ), providing evidence that this amino acid was able to evolve (from positively to negatively charged) without significantly compromising NatB acetyltransferase function.

Finally, whereas we provide mechanistic and in vivo evidence for a role of the NatB complex in neuronal survival, we have not been able to identify a target protein that would be sufficient to explain the $\mathrm{N}$-acetylation-dependent prevention of cell death. Two main reasons have made this endeavor impossible so far: (1) $60 \%$ or more of all proteins undergo cotranslational acetylation, and (2) this modification can only be detected by mass spectrometry making cell-specific analysis of developing fly tissues currently unfeasible. And although it would be highly desirable to identify such a key target protein, it is possible that this single protein does not exist but rather that the sheer number of non$N$-acetylated proteins does not allow for the cell to function and survive. Future studies and improved methodology will hopefully elucidate this point.

We conclude that Psidin provides an interesting regulator of neuronal development with key functions during neuronal survival and axon targeting. Thus, characterization of the in vivo role and the regulation of Psidin and its homologs could support research aimed at understanding regeneration and degeneration of the nervous system.

\section{References}

Ang LH, Chen W, Yao Y, Ozawa R, Tao E, Yonekura J, Uemura T, Keshishian $\mathrm{H}$, Hing $\mathrm{H}$ (2006) Lim kinase regulates the development of olfactory and neuromuscular synapses. Dev Biol 293:178-190.

Brennan CA, Delaney JR, Schneider DS, Anderson KV (2007) Psidin is re- 
quired in Drosophila blood cells for both phagocytic degradation and immune activation of the fat body. Curr Biol 17:67-72.

Brochtrup A, Hummel T (2011) Olfactory map formation in the Drosophila brain: genetic specificity and neuronal variability. Curr Opin Neurobiol 21:85-92.

Bugyi B, Carlier MF (2010) Control of actin filament treadmilling in cell motility. Annu Rev Biophys 39:449-470.

Campos-Ortega JA, Hartenstein V (1997) The embryonic development of Drosophila melanogaster, Ed 2. Berlin: Springer.

Cayirlioglu P, Kadow IG, Zhan X, Okamura K, Suh GS, Gunning D, Lai EC, Zipursky SL (2008) Hybrid neurons in a microRNA mutant are putative evolutionary intermediates in insect $\mathrm{CO} 2$ sensory systems. Science 319:1256-1260.

Davidson FF, Steller H (1998) Blocking apoptosis prevents blindness in Drosophila retinal degeneration mutants. Nature 391:587-591.

Dent EW, Gertler FB (2003) Cytoskeletal dynamics and transport in growth cone motility and axon guidance. Neuron 40:209-227.

Driessens MH, Hu H, Nobes CD, Self A, Jordens I, Goodman CS, Hall A (2001) Plexin-B semaphorin receptors interact directly with active Rac and regulate the actin cytoskeleton by activating Rho. Curr Biol 11:339-344

Dübendorfer A, Eichenberger-Glinz S (1980) Development and metamorphosis of larval and adult tissues of Drosophila in vitro. In: Invertebrate systems in vitro, pp 169-185. Amsterdam: Elsevier North Holland.

Evans IR, Renne T, Gertler FB, Nobes CD (2007) Ena/VASP proteins mediate repulsion from ephrin ligands. J Cell Sci 120:289-298.

Gonçalves-Pimentel C, Gombos R, Mihály J, Sánchez-SorianoN, Prokop A (2011) Dissecting regulatory networks of filopodia formation in a Drosophila growth cone model. PLoS One 6:e18340.

Gordon-Weeks PR (2004) Microtubules and growth cone function. J Neurobiol 58:70-83.

Green MR, Sambrook J (2012) Molecular cloning: a laboratory manual, Ed 4. Cold Spring Harbor, NY: Cold Spring Harbor Laboratory.

Hall A, Lalli G (2010) Rho and Ras GTPases in axon growth, guidance, and branching. Cold Spring Harb Perspect Biol 2:a001818.

Hartl M, Loschek LF, Stephan D, Siju KP, Knappmeyer C, Kadow IC (2011) A new Prospero and microRNA-279 pathway restricts $\mathrm{CO}_{2}$ receptor neuron formation. J Neurosci 31:15660-15673.

Huber AB, Kolodkin AL, Ginty DD, Cloutier JF (2003) Signaling at the growth cone: ligand-receptor complexes and the control of axon growth and guidance. Annu Rev Neurosci 26:509-563.

Kalil K, Dent EW (2005) Touch and go: guidance cues signal to the growth cone cytoskeleton. Curr Opin Neurobiol 15:521-526.

Kim JH, Cho A, Yin H, Schafer DA, Mouneimne G, Simpson KJ, Nguyen KV, Brugge JS, Montell DJ (2011) Psidin, a conserved protein that regulates protrusion dynamics and cell migration. Genes Dev 25:730-741.
Kuhn TB, Bamburg JR (2008) Tropomyosin and ADF/cofilin as collaborators and competitors. Adv Exp Med Biol 644:232-249.

Lowery LA, Van Vactor D (2009) The trip of the tip: understanding the growth cone machinery. Nat Rev Mol Cell Biol 10:332-343.

Matusek T, Gombos R, Szécsényi A, Sánchez-SorianoN, Czibula A, Pataki C, Gedai A, Prokop A, Raskó I, Mihály J (2008) Formin proteins of the DAAM subfamily play a role during axon growth. J Neurosci 28:13310-13319.

Morin X, Daneman R, Zavortink M, Chia W (2001) A protein trap strategy to detect GFP-tagged proteins expressed from their endogenous loci in Drosophila. Proc Natl Acad Sci U S A 98:15050-15055.

Newsome TP, Asling B, Dickson BJ (2000) Analysis of Drosophila photoreceptor axon guidance in eye-specific mosaics. Development 127:851-860.

Ng J, Luo L (2004) Rho GTPases regulate axon growth through convergent and divergent signaling pathways. Neuron 44:779-793.

Pak CW, Flynn KC, Bamburg JR (2008) Actin-binding proteins take the reins in growth cones. Nat Rev Neurosci 9:136-147.

Polevoda B, Sherman F (2003a) N-terminal acetyltransferases and sequence requirements for $\mathrm{N}$-terminal acetylation of eukaryotic proteins. J Mol Biol 325:595-622.

Polevoda B, Sherman F (2003b) Composition and function of the eukaryotic N-terminal acetyltransferase subunits. Biochem Biophys Res Commun 308:1-11.

Rodrigues V, Hummel T (2008) Development of the Drosophila olfactory system. Adv Exp Med Biol 628:82-101.

Sánchez-Soriano N, Gonçalves-PimentelC, Beaven R, Haessler U, OfnerZiegenfuss L, Ballestrem C, Prokop A (2010) Drosophila growth cones: a genetically tractable platform for the analysis of axonal growth dynamics. Dev Neurobiol 70:58-71.

Schneider I (1964) Differentiation of larval Drosophila eye-antennal discs in vitro. J Exp Zool 156:91-103.

Singer JM, Shaw JM (2003) Mdm20 protein functions with Nat3 protein to acetylate $\mathrm{Tpm} 1$ protein and regulate tropomyosin-actin interactions in budding yeast. Proc Natl Acad Sci U S A 100:7644-7649.

Starheim KK, Arnesen T, Gromyko D, Ryningen A, Varhaug JE, Lillehaug JR (2008) Identification of the human N(alpha)-acetyltransferase complex B (hNatB): a complex important for cell-cycle progression. Biochem J 415:325-331

Trost M, English L, Lemieux S, Courcelles M, Desjardins M, Thibault P (2009) The phagosomal proteome in interferon-gamma-activated macrophages. Immunity 30:143-154.

Vereshchagina N, Bennett D, SzöorB, Kirchner J, Gross S, Vissi E, WhiteCooper H, Alphey L (2004) The essential role of PP1beta in Drosophila is to regulate nonmuscle myosin. Mol Biol Cell 15:4395-4405.

Vosshall LB, Hansson BS (2011) A unified nomenclature system for the insect olfactory coreceptor. Chem Senses 36:497-498. 